州 


\title{
Akten zur Auswärtigen Politik der Bundesrepublik Deutschland
}

Herausgegeben im Auftrag des Auswärtigen Amts vom Institut für Zeitgeschichte

\author{
Hauptherausgeber \\ Hans-Peter Schwarz
}

Mitherausgeber

Helga Haftendorn, Klaus Hildebrand, Werner Link, Horst Möller und Rudolf Morsey

R. Oldenbourg Verlag München 1999 


\title{
Akten zur Auswärtigen Politik der Bundesrepublik Deutschland 1951
}

1. Januar bis 31. Dezember 1951

Wissenschaftlicher Leiter

Rainer A. Blasius

\author{
Bearbeiter \\ Matthias Jaroch
}

R. Oldenbourg Verlag München 1999 
Die Deutsche Bibliothek - CIP-Einheitsaufnahme

Akten zur auswärtigen Politik der Bundesrepublik Deutschland / hrsg. im Auftr. des Auswärtigen Amts vom Institut für

Zeitgeschichte. - München : Oldenbourg

1951. 1. Januar bis 31. Dezember 1951. - 1999

ISBN 3-486-56418-8

(C) 1999 Oldenbourg Wissenschaftsverlag GmbH, München Rosenheimer Straße 145, D-81671 München Internet: http://www.oldenbourg-verlag.de

Das Werk einschließlich aller Abbildungen ist urheberrechtlich geschützt. Jede Verwertung außerhalb der Grenzen des Urheberrechtsgesetzes ist ohne Zustimmung des Verlages unzulässig und strafbar. Dies gilt insbesondere für Vervielfältigungen, Übersetzungen, Mikroverfilmungen und die Einspeicherung und Bearbeitung in elektronischen Systemen.

Umschlaggestaltung: Dieter Vollendorf

Gedruckt auf säurefreiem, alterungsbeständigem Papier (chlorfrei gebleicht).

Gesamtherstellung: R. Oldenbourg Graphische Betriebe Druckerei GmbH, München

ISBN 3-486-56418-8 


\section{Inhalt}

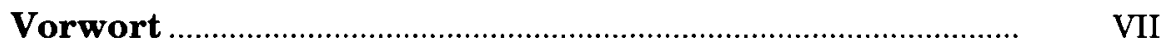

Vorbemerkungen zur Edition.................................................... VIII

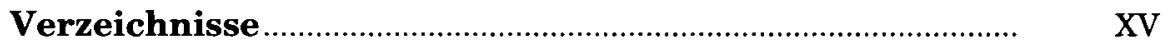

Dokumentenverzeichnis ................................................................. XVII

Literaturverzeichnis ..................................................................... XLVIII

Abkürzungsverzeichnis …………………………………..................... LVII

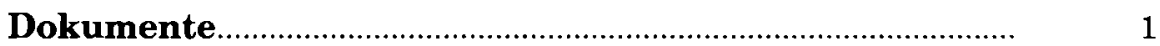

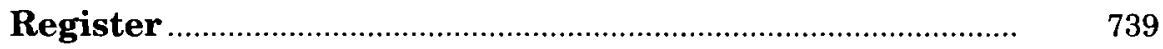

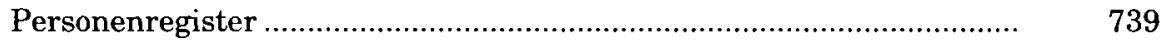

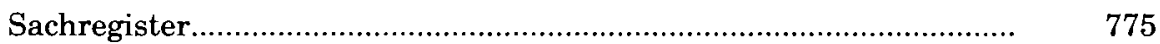

Organisationsplan des Auswärtigen Amts vom 10. Oktober 1951......... 817 



\section{Vorwort}

Mit den „Akten zur Auswärtigen Politik der Bundesrepublik Deutschland 1951“ wird die Publikation von Dokumenten aus dem Politischen Archiv des Auswärtigen Amts über die Jahre 1949 bis 1962 fortgesetzt. Der Band ergänzt die bereits 1989 unter meiner Verantwortung publizierte Edition „Adenauer und die Hohen Kommissare 1949-1951“.

Das Erscheinen des vorliegenden Bandes gibt Anlaß, allen an dem Werk Beteiligten zu danken. So gilt mein verbindlichster Dank dem Auswärtigen Amt, insbesondere dem Politischen Archiv sowie den Damen und Herren in den Referaten, die beim Deklassifizierungsverfahren zur Offenlegung der Dokumente beigetragen haben. In gleicher Weise zu danken ist dem Bundesarchiv für die Erlaubnis, einige Aufzeichnungen aus dem Nachlaß des Botschafters a.D. Herbert Blankenhorn und aus dem Bestand Bundeskanzleramt (B 136) einbeziehen zu können.

Besonderer Dank gebührt ferner den Kollegen im Herausgebergremium, die sich ihrer viel Zeit in Anspruch nehmenden Aufgabe in bewährter Kollegialität gewidmet haben. Ferner sei die tadellose Zusammenarbeit mit den zuständigen Persönlichkeiten und Gremien des Instituts für Zeitgeschichte dankbar hervorgehoben. Gedankt sei auch dem präzise arbeitenden Verlag R. Oldenbourg.

Das Hauptverdienst am Gelingen des Bandes gebührt dem Bearbeiter, Herrn Dr. Matthias Jaroch, zusammen mit dem Wissenschaftlichen Leiter, Herrn Dr. Rainer A. Blasius. Ihnen sei für die erbrachte Leistung nachdrücklichst gedankt. Ebenso haben wesentlich zur pünktlichen Fertigstellung der Edition beigetragen: Herr Dr. Daniel Kosthorst durch die Vorbereitung der Dokumentensammlung, Frau Dr. Ute Jancke durch Vorarbeiten für die Kommentierung, Frau Dr. Mechthild Lindemann durch die Bearbeitung von drei Monaten des Jahres 1951, Herr Dr. Joachim Wintzer durch die Erstellung des Personenregisters, Herr Dr. Martin Koopmann durch die Erstellung des Sachregisters und Herr Dr. Wolfgang Hölscher durch die Beratung bei der Herstellung des Umbruchs.

Die „Akten zur Auswärtigen Politik der Bundesrepublik Deutschland $1952^{\varkappa}$ befinden sich in Arbeit und werden voraussichtlich im Sommer 2000 vorliegen.

Bonn, den 1. Juli 1999

Hans-Peter Schwarz 


\section{Vorbemerkungen zur Edition}

Zu Beginn des Jahres 1951 lagen die Konsular- und Handelsbeziehungen der Bundesrepublik sowie die Verbindungen zur Alliierten Hohen Kommission (AHK) noch in der Zuständigkeit der am 7. Juni 1950 geschaffenen Dienststelle für Auswärtige Angelegenheiten im Bundeskanzleramt. Erst mit der „kleinen Revision" des Besatzungsstatuts am 6. März 1951 erteilten Frankreich, Großbritannien und die USA die Genehmigung, ein eigenes Außenministerium zu errichten. Der Bundesregierung wurde die Aufnahme diplomatischer Beziehungen mit anderen Staaten erlaubt, sofern dies vereinbar war mit der Sicherheit der Drei Mächte, mit den Rechten, die sie sich auf innen- und wirtschaftspolitischem Gebiet vorbehalten hatten, und mit den „Verpflichtungen der Besatzungsmächte in bezug auf Deutschland".

Bundespräsident Heuss ernannte am 15. März 1951 Bundeskanzler Adenauer zum Bundesminister des Auswärtigen. Am gleichen Tag wurde das Auswärtige Amt in Bonn errichtet und die Dienststelle für Auswärtige Angelegenheiten in das neue Bundesministerium überführt. Das Auswärtige Amt gliederte sich in sieben Abteilungen: Personal- und Verwaltungsabteilung, Politische Abteilung (zuständig für die AHK, allgemeine außenpolitische Fragen, Beziehungen zu internationalen Organisationen sowie Flüchtlings- und Kriegsgefangenenfragen), Länderabteilung (zuständig für die Lenkung der politischen und konsularischen Tätigkeit der Auslandsvertretungen), Handelspolitische Abteilung, Rechtsabteilung, Kulturabteilung und Protokoll. Der bisherige Leiter der Dienststelle für Auswärtige Angelegenheiten, Walter Hallstein, wurde am 2. April 1951 zum Staatssekretär des Auswärtigen Amts ernannt.

\section{Dokumentenauswahl}

Grundlage für die Fondsedition der „Akten zur Auswärtigen Politik der Bundesrepublik Deutschland 1951" (Kurztitel: AAPD 1951) sind die Bestände des Politischen Archivs des Auswärtigen Amts (PA/AA). Besonderes Gewicht wurde auf den Bestand der Politischen Abteilung gelegt. Angemessene Berücksichtigung fanden aber auch die anderen Abteilungen des Auswärtigen Amts, insbesondere die Länder- und Kulturabteilung. Für die Dokumentation der Endphase der Verhandlungen über den Schuman-Plan war der Bestand des im Juli 1950 eigens eingerichteten "Sekretariats für Fragen des Schuman-Plans“ von Bedeutung. Über die am 15. Februar 1951 beginnende Konferenz für die Organisation einer europäischen Armee, die nach Verabschiedung des Zwischenberichts vom 24. Juli 1951 in Konferenz für die Organisation einer Europäischen Verteidigungsgemeinschaft umbenannt wurde, gaben die Bestände des „Sekretariats für Fragen des Pleven-Plans“ sowie die Akten der "EVG-Delegation“ Aufschluß. Die Handakten des Leiters der Politischen Abteilung im Auswärtigen Amt, Herbert Blankenhorn, befinden sich zu einem Teil im PA/AA und zu einem anderen Teil im Bundesarchiv in Koblenz. Sie wurden ebenso ausgewertet wie einzelne Bestände aus dem Bundesarchiv-Militärarchiv in Freiburg und 
aus der Stiftung Bundeskanzler-Adenauer-Haus in Rhöndorf (Nachlaß Adenauer).

Die ausgewählten Dokumente ergänzen die bereits im Jahr 1989 publizierte Edition „Adenauer und die Hohen Kommissare 1949-1951“. Erstmals werden Schriftstücke veröffentlicht, die bisher als Verschlußsachen der Geheimhaltung unterworfen waren. Die VS-Bestände des PAAA waren uneingeschränkt zugänglich. Kopien der vom Auswärtigen Amt für diesen Band deklassifizierten Schriftstücke sind im PA/AA zugänglich (Bestand B 150).

Zur Dokumentation wesentlicher historischer Vorgänge wurden in Ausnahmefällen bereits an anderer Stelle veröffentlichte Schriftstücke von besonderer Bedeutung in die Auswahl aufgenommen.

\section{Dokumentenfolge}

Die 216 edierten Dokumente sind chronologisch geordnet und mit laufenden Nummern versehen. Bei differierenden Datumsangaben auf einem Schriftstück, z.B. im Falle abweichender maschinenschriftlicher und handschriftlicher Datierung, ist in der Regel das früheste Datum maßgebend. Mehrere Dokumente mit demselben Datum sind, soweit möglich, nach der Uhrzeit eingeordnet. Erfolgt eine Datierung lediglich aufgrund sekundärer Hinweise (z.B. aus Begleitschreiben, beigefügten Vermerken usw.), wird dies in einer Anmerkung ausgewiesen. Bei Aufzeichnungen über Gespräche oder Besprechungen ist das Datum des dokumentierten Vorgangs ausschlaggebend, nicht der meist spätere Zeitpunkt der Niederschrift.

\section{Dokumentenkopf}

Jedes Dokument beginnt mit einem halbfett gedruckten Dokumentenkopf, in dem wesentliche formale Angaben zusammengefaßt werden. Auf Dokumentennummer und -überschrift folgen in kleinerer Drucktype ergänzende Angaben, so rechts außen die Datumsangabe. Links außen wird, sofern vorhanden, das Geschäftszeichen des edierten Schriftstücks einschließlich des Geheimhaltungsgrads (zum Zeitpunkt der Entstehung) wiedergegeben. Das Geschäftszeichen, das Rückschlüsse auf den Geschäftsgang zuläßt und die Ermittlung zugehörigen Aktenmaterials ermöglicht, besteht in der Regel aus der Kurzbezeichnung der ausfertigenden Arbeitseinheit sowie aus weiteren Elementen wie dem inhaltlich definierten Aktenzeichen, der Tagebuchnummer einschließlich verkürzter Jahresangabe und gegebenenfalls dem Geheimhaltungsgrad. Bei Abweichungen von diesem Schema folgt die Angabe der jeweils auf dem Dokument vorgefundenen Eintragung. Dokumentennummer, verkürzte Überschrift und Datum finden sich auch im Kolumnentitel über dem Dokument.

Den Angaben im Dokumentenkopf, vor allem der Überschrift, läßt sich die Art des jeweiligen Dokuments entnehmen. Aufzeichnungen sind eine in der Edition besonders häufig vertretene Dokumentengruppe. Der Verfasser wird jeweils in der Überschrift benannt, auch dann, wenn er sich nur indirekt erschließen läßt. Letzteres wird in einer Anmerkung vermerkt. Läßt sich ein Verfasser we- 
der mittelbar noch unmittelbar feststellen, wird die ausführende Arbeitseinheit (Abteilung, Referat oder Delegation) angegeben.

Breiten Raum nehmen ferner Gesprächsaufzeichnungen sowie Niederschriften über Besprechungen und Konferenzen ein. Sie werden als solche in der Überschrift gekennzeichnet und chronologisch nach dem Gesprächs-, Besprechungsoder Konferenzdatum eingeordnet, während Verfasser und Datum der Niederschrift - sofern ermittelbar - in einer Anmerkung ausgewiesen sind.

Eine wichtige Dokumentengruppe sind darüber hinaus Schreiben, erkennbar jeweils an der Nennung von Absender und Empfänger. Dazu gehören insbesondere die Schreiben des Bundeskanzlers an die Hohen Kommissare, die entweder an einen der Hohen Kommissare allein oder an den im monatlichen Wechsel amtierenden Geschäftsführenden Vorsitzenden der AHK gerichtet wurden.

Eine weitere Gruppe von Dokumenten bildet der Schriftverkehr mit den seit Juni 1950 errichteten Generalkonsulaten im Ausland. Diese informierten in der Regel mit Schriftberichten, seltener auch mit fernschriftlich übermittelten Drahtberichten. Auch bei dieser Dokumentengruppe wird in der Überschrift der Verfasser genannt. Als Empfänger werden die Dienststelle für Auswärtige Angelegenheiten bzw. das Auswärtige Amt angegeben, sofern kein bestimmter Adressat erkennbar ist. Bei Schriftberichten werden, soweit vorhanden, im unteren Teil des Dokumentenkopfes links das Geschäftszeichen und die Nummer des Berichts, bei Drahtberichten ferner der Grad der Dringlichkeit angegeben. Rechts davon befinden sich Datum und gegebenenfalls Uhrzeit der Aufgabe bzw. der Ankunft, jeweils in Ortszeit. Ein Ausstellungsdatum wird nur dann angegeben, wenn es vom Datum der Aufgabe abweicht.

Die wenigen Dokumente, die sich keiner der beschriebenen Gruppen zuordnen lassen, sind aufgrund individueller Überschriften zu identifizieren.

Die Überschrift bei allen Dokumenten enthält die notwendigen Angaben zum Ausstellungs-, Absende- oder Empfangsort bzw. zum Ort des Gesprächs oder der Besprechung. Erfolgt keine besondere Ortsangabe, ist Bonn stillschweigend zu ergänzen. Hält sich der Verfasser oder Absender eines Dokuments nicht an seinem eigentlichen Dienstort auf, wird der Ortsangabe ein „z.Z." vorangesetzt.

Bei den edierten Schriftstücken handelt es sich in der Regel jeweils um die erste Ausfertigung oder - wie etwa bei den Drahtberichten - um eines von mehreren gleichrangig nebeneinander zirkulierenden Exemplaren. Statt der Erstausfertigung mußten gelegentlich ein Durchdruck, eine Abschrift, eine Ablichtung oder ein vervielfältigtes Exemplar (Matrizenabzug) herangezogen werden. Eine besondere Gruppe bilden einige Schreiben, deren Wortlaut nur in der handschriftlich korrigierten Fassung des Konzepts vorliegt. Ein entsprechender Hinweis findet sich in einer Anmerkung. In wenigen Fällen sind Entwürfe abgedruckt und entsprechend in den Überschriften kenntlich gemacht.

\section{Dokumententext}

Unterhalb des Dokumentenkopfes folgt - in normaler Drucktype - der Text des jeweiligen Dokuments, einschließlich des Betreffs, der Anrede und der Unterschrift. Falls die Textvorlage eine inhaltlich substantielle Überschrift aufweist, wird diese mitabgedruckt. Die Dokumente werden in der Regel ungekürzt pub- 
liziert. Sofern in Ausnahmefällen Auszüge veröffentlicht werden, ist dies in einer Anmerkung vermerkt. Textergänzungen der Bearbeiter stehen in eckigen Klammern.

Offensichtliche Schreib- und Interpunktionsfehler werden stillschweigend korrigiert. Eigentümliche Schreibweisen bleiben nach Möglichkeit erhalten; im Bedarfsfall wird jedoch vereinheitlicht bzw. modernisiert. Dies trifft teilweise auch auf fremdsprachige Orts- und Personennamen zu, deren Schreibweise nach den im Auswärtigen Amt gebräuchlichen Regeln wiedergegeben wird.

Selten vorkommende und ungebräuchliche Abkürzungen in der Textvorlage werden aufgelöst. Typische Abkürzungen von Institutionen, Parteien etc. werden allerdings übernommen. Hervorhebungen in der Textvorlage, also etwa maschinenschriftliche Unterstreichungen oder Sperrungen, werden nicht wiedergegeben. Der Kursivdruck dient dazu, bei Gesprächsaufzeichnungen die Sprecher voneinander abzuheben. Im äußeren Aufbau (Absätze, Überschriften usw.) folgt das Druckbild weitgehend der Textvorlage.

Mit Ausnahme der dem Namen hinzugefügten Dienstbezeichnung, die der Überschrift eines Dokuments zu entnehmen ist, wird eine Unterschriftsformel vollständig wiedergegeben. Ein handschriftlicher Namenszug ist nicht besonders gekennzeichnet, eine Paraphe mit Unterschriftscharakter aufgelöst (mit Nachweis in einer Anmerkung). Findet sich auf einem Schriftstück der Name zusätzlich maschinenschriftlich vermerkt, bleibt dies unerwähnt. Ein maschinenschriftlicher Name, dem ein „gez." vorangestellt ist, wird entsprechend übernommen; fehlt in der Textvorlage der Zusatz "gez.", wird er in eckigen Klammern ergänzt. Weicht das Datum der Paraphe vom Datum des Schriftstückes ab, wird dies in der Anmerkung ausgewiesen.

Unter dem Dokumententext wird die jeweilige Fundstelle des Schriftstückes in halbfetter Schrifttype nachgewiesen. Bei Dokumenten aus dem PA/AA wird auf die Angabe des Archivs verzichtet und nur der jeweilige Bestand mit Bandnummer genannt. Dokumente aus VS-Beständen sind mit der Angabe „VS-Bd.“ versehen. Bei Dokumenten anderer Herkunft werden Archiv und Bestandsbezeichnung angegeben. Liegt ein Schriftstück bereits veröffentlicht vor, so wird dies in einer gesonderten Anmerkung nach der Angabe der Fundstelle ausgewiesen. Für den Fall, daß das Dokument mehrfach publiziert wurde, wird lediglich eine leicht zugängliche Publikation genannt.

\section{Kommentierung}

In Ergänzung zum Dokumentenkopf enthalten die Anmerkungen formale Hinweise und geben Auskunft über wesentliche Stationen im Geschäftsgang. Angaben technischer Art, wie z. B. Registraturvermerke, werden nur bei besonderer Bedeutung erfaßt. Wesentlich ist dagegen die Frage, welche Beachtung das jeweils edierte Dokument auf den verschiedenen Ebenen der Dienststelle für Auswärtige Angelegenheiten bzw. des Auswärtigen Amts gefunden hat. Dies läßt sich an den Paraphen maßgeblicher Akteure sowie an den - überwiegend handschriftlichen - Weisungen, Bemerkungen oder auch Reaktionen in Form von Frage- oder Ausrufungszeichen ablesen, die auf dem Schriftstück selbst oder auf zugehörigen Begleitschreiben und -vermerken zu finden sind. Die 
diesbezüglichen Merkmale sowie damit in Verbindung stehende Hervorhebungen (Unterstreichungen oder Anstreichungen am Rand) werden in Anmerkungen nachgewiesen. Auf den Nachweis sonstiger An- oder Unterstreichungen wird verzichtet. Abkürzungen in handschriftlichen Passagen werden unter Kennzeichnung durch eckige Klammern aufgelöst.

In den im engeren Sinn textkritischen Anmerkungen werden nachträgliche Korrekturen oder textliche Änderungen festgehalten, sofern Konzipient und Verfasser nicht identisch sind. Unwesentliche Textverbesserungen sind hiervon ausgenommen. Ferner wird auf einen systematischen Vergleich der Dokumente mit Entwürfen ebenso verzichtet wie auf den Nachweis der in der Praxis üblichen Einarbeitung von Textpassagen in eine spätere Aufzeichnung.

Die Kommentierung soll den historischen Zusammenhang der edierten Dokumente in ihrer zeitlichen und inhaltlichen Abfolge sichtbar machen, weiteres Aktenmaterial und anderweitiges Schriftgut nachweisen, das unmittelbar oder mittelbar angesprochen wird, sowie Ereignisse oder Sachverhalte näher erläutern, die dem heutigen Wissens- und Erfahrungshorizont ferner liegen und aus dem Textzusammenhang heraus nicht oder nicht hinlänglich zu verstehen sind. Das Auffinden von Dokumenten zu einem bestimmten thematischen Schwerpunkt ist mit Hilfe des Sachregisters möglich.

Besonderer Wert wird bei der Kommentierung darauf gelegt, daß die Dokumente durch Bezugsstücke aus der zugehörigen Aktenüberlieferung oder gegebenenfalls bereits vorliegenden Editionen und Dokumentationen erläutert werden. Zitate und inhaltliche Wiedergaben sollen die Entscheidungsprozesse erhellen. Dadurch werden Vorarbeiten für eine vertiefende Erschließung der Bestände des PA/AA geleistet.

Findet in einem Dokument veröffentlichtes Schriftgut Erwähnung - etwa Abkommen, Gesetze, Reden oder Presseberichte -, so wird die Fundstelle in einer Anmerkung nach Möglichkeit genauer spezifiziert. Auszüge aus den Bezugsstücken oder inhaltliche Zusammenfassungen sollen zum Verständnis der Dokumente beitragen. Bei Anmerkungen oder Anmerkungsteilen, deren Zweck die knappe Erläuterung eines Sachverhalts oder Ereignisses ist, erfolgen keine systematischen Hinweise auf archivalische oder veröffentlichte Quellen. Sekundärliteratur wird generell nicht in die Kommentierung aufgenommen.

Angaben wie Dienstbezeichnung, Dienststellung, Funktion, Dienstbehörde und Nationalität dienen der eindeutigen Identifizierung der in der Kommentierung vorkommenden Personen. Bei Bundesministern erfolgt ein Hinweis zum jeweiligen Ressort nur im Personenregister. Eine im Dokumententext lediglich mit ihrer Funktion genannte Person wird nach Möglichkeit in einer Anmerkung namentlich nachgewiesen. Davon ausgenommen sind der jeweilige Bundespräsident und der Bundeskanzler bzw. der Bundesminister des Auswärtigen.

Die Bezeichnung einzelner Staaten wird so gewählt, daß Verwechslungen ausgeschlossen sind. Als Kurzform für die Deutsche Demokratische Republik kommen in den Dokumenten die Begriffe SBZ oder DDR vor und werden so wiedergegeben. Der in der Forschung üblichen Praxis folgend, wird jedoch in der Kommentierung, den Verzeichnissen sowie den Registern der Begriff DDR verwendet. Das Adjektiv „deutsch" findet nur bei gesamtdeutschen Belangen oder dann Verwendung, wenn eine eindeutige Zuordnung gegeben ist. Der 
westliche Teil von Berlin wird als Berlin (West), der östliche Teil der Stadt als Ost-Berlin bezeichnet.

Für häufig benutzte Publikationen wie Editionen, Geschichtskalender und Memoiren werden Kurztitel oder Kurzformen eingeführt, die sich über ein entsprechendes Verzeichnis auflösen lassen. Der Platzersparnis dienen ebenfalls die Rückverweise auf bereits an anderer Stelle ausgeführte Anmerkungen. Häufig genannte Verträge oder Gesetzestexte werden nur bei der Erstnennung nachgewiesen und lassen sich über das Sachregister erschließen.

Wie bei der Wiedergabe der Dokumente finden auch in den Anmerkungen die im Auswärtigen Amt gebräuchlichen Regeln für die Transkription fremdsprachlicher Namen und Begriffe Anwendung. Bei Literaturangaben in russischer Sprache wird die im wissenschaftlichen Bereich übliche Transliterierung durchgeführt.

\section{Verzeichnisse}

Das Dokumentenverzeichnis ist chronologisch angelegt. Es bietet zu jedem Dokument folgende Angaben: Die halbfett gedruckte Dokumentennummer, Datum und Überschrift, die Fundseite sowie eine inhaltliche Kurzübersicht, in der hauptsächlich die angesprochenen Themen benannt werden.

Das Literaturverzeichnis enthält nur solche Publikationen, die häufig zur Kommentierung herangezogen und mit Kurztiteln oder Kurzformen versehen wurden. Diese sind alphabetisch geordnet und werden unter Angabe der notwendigen bibliographischen Daten aufgelöst.

Das Abkürzungsverzeichnis führt die im Dokumententeil vorkommenden Abkürzungen auf, insbesondere von Firmen, Organisationen, Parteien und Dienstbezeichnungen sowie sonstige im diplomatischen Schriftverkehr übliche Abbreviaturen. Nicht aufgenommen werden geläufige Abkürzungen wie „z. B.“, „d.h.“, „m. E.“, „u.U.“ und „usw.“ sowie Abkürzungen, die bereits in einer Anmerkung erläutert sind.

\section{Register und Organisationsplan}

Im Personenregister werden in der Edition vorkommende Personen unter Nennung derjenigen politischen, dienstlichen oder beruflichen Funktionen aufgeführt, die im inhaltlichen Zusammenhang der Dokumente wesentlich sind. Das Sachregister ermöglicht einen thematisch differenzierten Zugriff auf die einzelnen Dokumente. Näheres ist den Hinweisen zur Benutzung des jeweiligen Registers auf S. 740 und S. 776 zu entnehmen.

Der Organisationsplan vom 10. Oktober 1951 zeigt die Struktur des Auswärtigen Amts und informiert über die Namen der Leiter der jeweiligen Arbeitseinheiten. 

Verzeichnisse 



\section{Dokumentenverzeichnis}

$\mathbf{1}$

02.01. Gespräch des Bundeskanzlers Adenauer mit dem amerikanischen Hohen Kommissar McCloy

Themen sind die amerikanische Verteidigungspolitik, der sowjetische Vorschlag zur Einberufung einer Außenministerkonferenz der Vier Mächte, ein Verteidigungsbeitrag der Bundesrepublik, die Errichtung einer Bundespolizei und die Anerkennung der deutschen Auslandsschulden.

\subsection{Aufzeichnung des Vortragenden Legationsrats} Dittmann

Dittmann berichtet von einem Gespräch mit dem iranischen Vizekonsul Logman-Adham über die Errichtung eines Generalkonsulats in Teheran.

05.01. Gespräch des Beauftragten des Bundeskanzlers, Blank, mit dem amerikanischen Stellvertretenden Hohen

Kommissar Hays

Blank und Hays erörtern Fragen eines Verteidigungsbeitrags der Bundesrepublik.

06.01. Generalkonsul Hausenstein, Paris, an die Dienststelle für Auswärtige Angelegenheiten

Hausenstein berichtet über ein Gespräch des Vortragenden Legationsrats von Kessel mit dem Mitarbeiter im französischen Außenministerium, Sauvagnargues. Im Mittelpunkt standen die Tagesordnung für eine von der UdSSR vorgeschlagene Außenministerkonferenz der Vier Mächte und ein Verteidigungsbeitrag der Bundesrepublik.

Steg informiert über das Verhältnis Israels zur Bundesrepublik, insbesondere hinsichtlich einer Regelung der Ansprüche auf Wiedergutmachung. Kommission

In der ersten Besprechung mit Vertretern der AHK auf dem Petersberg über einen Verteidigungsbeitrag der Bundesrepublik wird erörtert, unter welchen Bedingungen, in welcher Form und in welcher Stärke die Bundesrepublik an der westlichen Verteidigung beteiligt werden könne.
S. 3

S. 10

S. 11

S. 19

S. 27 
7

12.01. Aufzeichnung des Vortragenden Legationsrats Riesser, New York

Riesser berichtet von Gesprächen mit Diplomaten in Washington. Thematisiert wurden die völkerrechtliche Beendigung des Kriegszustands mit Deutschland, ein Verteidigungsbeitrag der Bundesrepublik, Gerüchte über eine Ablösung des amerikanischen Außenministers Acheson sowie die Einschätzung der Bedrohung Europas durch die UdSSR.

8

13.01. Aufzeichnung des Vortragenden Legationsrats Riesser, New York

Riesser berichtet von einem Gespräch mit dem ehemaligen Präsidenten Hoover über das Problem der Verteidigung Europas.

$\mathbf{9}$

13.01. Instruktionen für die Delegation bei der Konferenz über den Schuman-Plan in Paris (Entwurf)

Der Entwurf enthält Richtlinien zu einzelnen Bestimmungen eines Vertrags zur Bildung einer Europäischen Gemeinschaft für Kohle und Stahl sowie zu einer Übergangsregelung.

10

15.01. Ressortbesprechung

In der Ressortbesprechung unter Leitung des Bundeskanzlers Adenauer werden mit den Verhandlungen über den SchumanPlan zusammenhängende Themen erörtert, besonders die Verbundwirtschaft von Kohle und Eisen sowie die Auflösung der Deutschen Kohle-Verkaufsorganisation.

11

15.01. Gespräch des Bundeskanzlers Adenauer mit dem

Parlamentarischen Staatssekretär im britischen

Außenministerium, Davies

Themen sind die Integration der Bundesrepublik in das westliche Verteidigungssystem, die militärische Bedrohung Europas durch die UdSSR und die Anerkennung der deutschen Auslandsschulden.

12

15.01. Aufzeichnung des Staatssekretärs Hallstein

Hallstein informiert über ein Gespräch mit dem amerikanischen Hohen Kommissar McCloy zu Fragen der Neuordnung des Kohlenbergbaus sowie der Eisen- und Stahlindustrie.

13

16.01. Aufzeichnung des Oberregierungsrats Ostermann von Roth

Ostermann von Roth faßt die zweite Besprechung mit Vertretern der AHK auf dem Petersberg über einen Verteidigungsbeitrag der Bundesrepublik zusammen. Der Beauftragte des Bundeskanzlers, Blank, legte dar, in welchen Phasen sich die Aufstellung eines Truppenkontingents vollziehen könne. 
Ophüls hält ein Gespräch mit dem Generalsekretär der Konferenz über den Schuman-Plan in Paris, Valérie, fest. Thema war die Aufhebung besatzungsrechtlicher Restriktionen beim Inkrafttreten des Schuman-Plans.

Kordt informiert über ein Gespräch mit dem irischen Generalkonsul Belton. Im Mittelpunkt stand die Aufnahme diplomatischer Beziehungen.

Kaufmann berichtet von den unterschiedlichen Auffassungen der Drei Mächte bei der Abfassung der Präambel zu dem Gesetz über die Aufhebung solcher Bestimmungen in der Gesetzgebung der Bundesrepublik, die noch auf dem völkerrechtlichen Kriegszustand beruhen. (Entwurf)

In dem Entwurf wird die Neuregelung der Beziehungen zwischen der Bundesrepublik und den Drei Mächten nach Ablösung des Besatzungsstatuts angekündigt.

Schmid erläutert die Einwände des Ausschusses des Bundestages für das Besatzungsstatut und auswärtige Angelegenheiten gegen den mit der AHK vereinbarten Entwurf eines Notenwechsels über die Anerkennung der deutschen Auslandsschulden. Roth

Ostermann von Roth faßt die dritte Besprechung mit Vertretern der AHK auf dem Petersberg über einen Verteidigungsbeitrag der Bundesrepublik zusammen, in der rechtliche Voraussetzungen für den Einsatz von Soldaten der Bundesrepublik erörtert wurden. Die Vorstellungen der Bundesregierung über die Zusammensetzung der Einheiten sowie über die Gliederung und Stärke eines modernen Kampfverbandes wurden erläutert.

Pfeiffer berichtet von einem Gespräch mit dem belgischen Außenminister van Zeeland über den Prozeß gegen den ehemaligen Militärbefehlshaber in Belgien und Nordfrankreich, Alexander von Falkenhausen, und über Grenzfragen. 
21

02.02. Besprechung mit Vertretern der Alliierten Hohen Kommission

In der vierten Besprechung mit Vertretern der AHK auf dem Petersberg über einen Verteidigungsbeitrag der Bundesrepublik nimmt die AHK zu den bisherigen Vorschlägen der Bundesregierung Stellung, jedoch nicht zu Stärke, Organisation und Zusammensetzung von Truppenkontingenten der Bundesrepublik. Der Beauftragte des Bundeskanzlers, Blank, äußert sich zu Ausrüstung und Bewaffnung. an die Dienststelle für Auswärtige Angelegenheiten

Brentano berichtet von einem Gespräch mit dem italienischen Außenminister Sforza über Fragen der europäischen Einigung und Sicherheit.

06.02. Generalkonsul I. Klasse Du Mont, Amsterdam, an die Dienststelle für Auswärtige Angelegenheiten

Du Mont informiert über die Besorgnis im niederländischen Außenministerium, daß die Bundesregierung auf Angebote der DDR zur Wiedervereinigung Deutschlands eingehen könne.

06.02. Generalkonsul I. Klasse Du Mont, Amsterdam, an die Dienststelle für Auswärtige Angelegenheiten

Du Mont berichtet von den Überlegungen der niederländischen Regierung, mit der Bundesregierung in Verhandlungen über die Grenzfrage einzutreten.

07.02. Generalkonsul I. Klasse Clemens von Brentano, Rom, an die Dienststelle für Auswärtige Angelegenheiten

Brentano hält ein Gespräch mit Ministerpräsident de Gasperi über Fragen der europäischen Verteidigung fest.

12.02. Vortragender Legationsrat Dittmann an Vortragenden Legationsrat von Kessel, Paris

Dittmann informiert Kessel über die neuesten Entwicklungen in der Diskussion um einen Verteidigungsbeitrag der Bundesrepublik. Hallstein, z.Z. Paris

Blankenhorn teilt Hallstein dessen Ernennung zum Delegationsleiter bei der Konferenz für die Organisation einer europäischen Armee mit. Zum militärischen Sachverständigen wird Oberstleutnant a.D. de Maizière berufen.

S. 85

S. 90 

Kommission

In der fünften Besprechung mit Vertretern der AHK auf dem Petersberg über einen Verteidigungsbeitrag der Bundesrepublik werden Fragen der Dislozierung sowie der Stärke von Truppenkontingenten der Bundesrepublik behandelt.

16.02. Aufzeichnung des Vortragenden Legationsrats Dittmann

Dittmann informiert über den Wunsch des amerikanischen Hohen Kommissars McCloy nach einer Aussprache mit Bundeskanzler Adenauer über die Schwierigkeiten, die bei den Verhandlungen über den Schuman-Plan und bei der Umsetzung des Gesetzes Nr. 27 der AHK aufgetreten sind.

Böker informiert über seine Reise in die USA vom 27. Januar bis 15. Februar 1951. Paris

De Maizière analysiert die militärischen Bestimmungen des französischen Memorandums vom 15. Februar 1951 über die Organisation einer europäischen Armee. Adenauer

Reuter äußert sich zur staatsrechtlichen Einbeziehung von Berlin (West) in die Bundesrepublik, zur Abgrenzung der Finanzverantwortlichkeit, zur Verlegung von Bundesbehörden und zum Zugang nach Berlin (West).

In der Ressortbesprechung wird insbesondere das französische Memorandum vom 15. Februar 1951 über die Organisation einer europäischen Armee erörtert. Dienststelle für Auswärtige Angelegenheiten

Marchtaler faßt die Sitzung der Delegationschefs bei der Konferenz für die Organisation einer europäischen Armee zusammen. Der belgische Delegationsleiter, Baron de Gruben, äußerte Bedenken gegenüber dem französischen Kampfgruppen-Konzept. 
35

26.02. Gespräch des Bundeskanzlers Adenauer mit dem italienischen Außenhandelsminister Lombardo

Adenauer und Lombardo erörtern Fragen der Eingliederung der Bundesrepublik in das westeuropäische Staatensystem, die bilateralen Wirtschaftsbeziehungen sowie die Fortführung der amerikanischen Wirtschaftshilfe.

36

27.02. Ressortbesprechung

Erörtert werden organisatorische Fragen bei den Verhandlungen über eine europäische Armee in Paris und die Stellungnahme zum französischen Kampfgruppen-Konzept.

37

27.02. Legationsrat I. Klasse von Marchtaler, Paris, an Staatssekretär Hallstein

Marchtaler unterrichtet Hallstein von einem Gespräch mit dem amerikanischen Botschafter in Paris, Bruce, über Fragen einer europäischen Armee.

38

28.02 Aufzeichnung des Vortragenden Legationsrats Dittmann

Dittmann informiert über ein Gespräch mit dem Politischen Berater im amerikanischen Hochkommissariat, Reber, anläßlich der Übergabe eines Aide-mémoire der AHK zur Revision des Besatzungsstatuts.

39

02.03. Aufzeichnung des Oberregierungsrats Ostermann von Roth

Ostermann von Roth faßt die sechste Besprechung mit Vertretern der AHK auf dem Petersberg über einen Verteidigungsbeitrag der Bundesrepublik zusammen, in der die AHK die Auffassung zur Rechtsstellung von Truppenkontingenten der Bundesrepublik im Kriegsfall darlegte. Die Delegation der Bundesrepublik äußerte sich zu Stärke und Zusammensetzung von militärischen Einheiten einer internationalen Armee. Bundeskanzler Adenauer

Pfeiffer informiert Adenauer über einen Gedankenaustausch mit dem amerikanischen Botschafter in Brüssel, Murphy. Im Mittelpunkt stand die Einstellung des amerikanischen Hohen Kommissars McCloy gegenüber der Bundesrepublik.
S. 132

S. 136

S. 142

S. 145

S. 149

S. 150 

amerikanischen Hohen Kommissar McCloy

Themen sind die Verhandlungen über die Organisation einer europäischen Armee, die Neuordnung im Kohlenbergbau sowie in der Eisen- und Stahlindustrie, die Kürzung amerikanischer Wirtschaftshilfe und Getreidelieferungen sowie die Revisionsverfahren gegen Deutsche, die wegen Kriegsverbrechen von amerikanischen Militärgerichten verurteilt wurden.

Adenauer dankt Kirkpatrick für die britische Zusage, die Insel Helgoland bis zum 1. März 1952 freizugeben, und sagt Unterstützung bei der Zuweisung von Ersatzzielen für die britische Luftwaffe zu. Bundeskanzler Adenauer

Krekeler berichtet von einem Gespräch mit dem Sonderberater des amerikanischen Präsidenten, Harriman, über die Eingliederung der Bundesrepublik in das westliche Verteidigungssystem. französischen Hohen Kommissar François-Poncet

Themen sind das Mitbestimmungsrecht in Kohlenbergwerken, die Vorkonferenz der stellvertretenden Außenminister der Vier Mächte in Paris und die vorgesehene Erklärung des Bundeskanzlers Adenauer vor dem Bundestag zu gesamtdeutschen Wahlen.

Hallstein leitet eine Mitteilung des französischen Hohen Kommissars François-Poncet an Bundeskanzler Adenauer zur Saarfrage weiter.

Themen sind die Stellungnahme auf der Konferenz für die Organisation einer europäischen Armee in Paris zum französischen Kampfgruppen-Konzept, die Beschlüsse der NATO-Ministerratstagung vom 18./19. Dezember 1950 sowie die französische Haltung zu einem Verteidigungsbeitrag der Bundesrepublik. die Organisation einer europäischen Armee in Paris

Das weitere Vorgehen bei der Konferenz für die Organisation einer europäischen Armee wird festgelegt. 
48

06.03. Bundeskanzler Adenauer an den Geschäftsführenden Vorsitzenden der Alliierten Hohen Kommission, François-Poncet

Adenauer informiert François-Poncet über die Anerkennung der deutschen Auslandsschulden durch die Bundesregierung.

49

07.03. Vortragender Legationsrat von Kessel, Paris, an die Dienststelle für Auswärtige Angelegenheiten

Kessel leitet Informationen über den Verlauf der Vorkonferenz der stellvertretenden Außenminister der Vier Mächte weiter.

50

09.03. Vortragender Legationsrat a. D. Roediger, Paris, an Staatssekretär Hallstein

Roediger teilt mit, daß sich die französische Delegation bei der Konferenz für die Organisation einer europäischen Armee in der Frage der Mindeststärke homogener nationaler Einheiten gesprächsbereit gezeigt habe.

51

09.03. Aufzeichnung des Oberregierungsrats Ostermann von Roth

Ostermann von Roth faßt Mitteilungen des Mitarbeiters im französischen Hochkommissariat, Cheysson, über die ersten drei Sitzungen der Vorkonferenz der stellvertretenden Außenminister der Vier Mächte in Paris zusammen.

13.03. Aufzeichnung des Referenten Böker, z.Z. Paris

Böker berichtet über ein Gespräch mit dem Mitarbeiter der ECA in Paris, Riddleberger, zu Fragen einer europäischen Armee und eines Verteidigungsbeitrags der Bundesrepublik.

Böker informiert über ein Gespräch mit dem Mitarbeiter der ECA in Paris, Riddleberger, der sich zum Verhältnis zwischen einer europäischen Armee und der NATO äußerte.

54

15.03 Bundeskanzler Adenauer an den Geschäftsführenden Vorsitzenden der Alliierten Hohen Kommission, François-Poncet

Adenauer teilt François-Poncet mit, daß er die Leitung des neu errichteten Auswärtigen Amts übernommen habe.
S. 176

S. 181

Kielmansegg faßt die siebte Besprechung mit Vertretern der AHK auf dem Petersberg über einen Verteidigungsbeitrag der Bundesrepublik zusammen. Erörtert wurden die Kosten des Aufbaus von Truppenkontingenten in einer Gesamtstärke von 250000 Mann. 

Dittmann

Dittmann berichtet über ein Gespräch des Bundeskanzlers Adenauer mit dem dänischen Außenminister Kraft. Im Mittelpunkt stand die Haltung der Bundesregierung zur Kieler Erklärung vom 26. September 1949 und die Herstellung bilateraler Beziehungen.

Dittmann

Dittmann hält ein Gespräch mit dem französischen Hohen Kommissar François-Poncet fest. Themen waren die Rundfunkansprache des Bundeskanzlers Adenauer zur Paraphierung des Schuman-Plans, eine Rede des SPD-Vorsitzenden Schumacher in Kiel und Äußerungen des Bundesministers Kaiser in Salzburg.

Lahusen erörtert, ob als Auslandsvertretungen ausschließlich Gesandtschaften oder auch Botschaften errichtet werden sollten. an das Auswärtige Amt

Brentano berichtet von einem Gespräch mit Ministerpräsident de Gasperi über dessen Unterredungen mit britischen und französischen Staatsmännern und über einen Besuch des Bundeskanzlers Adenauer in Italien.

60 28.03. Aufzeichnung des Legationsrats I. Klasse Salat

Salat informiert über die Bemühungen der Bundesregierung um Aufnahme in die UNESCO und über das Beitrittsverfahren.

Adenauer und Henderson erörtern Fragen der europäischen Einigung. Marchtaler, Paris

Marchtaler berichtet über die französische Reaktion auf die $\mathbf{P a}$ raphierung des Schuman-Plans durch die Bundesregierung, die Saarfrage, die bilateralen Beziehungen und die Vorkonferenz der stellvertretenden Außenminister der Vier Mächte. 
Einvernehmen wird darüber erzielt, daß wegen der bevorstehenden Wahlen zur französischen Nationalversammlung neue Vorschläge der Delegation der Bundesrepublik auf der Konferenz für die Organisation einer europäischen Armee in Paris vorerst zurückgestellt werden.

Kielmansegg faßt die achte Besprechung mit Vertretern der AHK auf dem Petersberg über einen Verteidigungsbeitrag der Bundesrepublik zusammen. Thematisiert wurden die Errichtung eines Verteidigungsministeriums und einer Generalinspektion sowie die Luftverteidigung.

Adenauer erinnert an die Sicherheitsgarantie der Drei Mächte zum Schutz der Bundesrepublik und von Berlin (West) und bittet um Truppenverstärkungen im Bundesgebiet.

Ophüls skizziert die Positionen auf der Konferenz über den Schuman-Plan in Paris zu einzelnen Artikeln des Vertrags über die Gründung der Europäischen Gemeinschaft für Kohle und Stahl. Vorsitzenden der Alliierten Hohen Kommission, Kirkpatrick

Adenauer bittet die AHK um Zustimmung zur Aufnahme diplomatischer oder konsularischer Beziehungen mit einer Reihe von Staaten.

Adenauer unterbreitet Schuman Vorschläge zur Behandlung des Saarproblems im Rahmen des Vertrags über die Gründung der Europäischen Gemeinschaft für Kohle und Stahl.

Roediger berichtet von seinem Vortrag bei Bundeskanzler Adenauer über den Verlauf der Verhandlungen bei der Konferenz für die Organisation einer europäischen Armee und von dem anschließenden Gespräch zwischen Adenauer und dem französischen Delegationsleiter Alphand. 
Kielmansegg faßt die neunte Besprechung mit Vertretern der AHK auf dem Petersberg über einen Verteidigungsbeitrag der Bundesrepublik zusammen. Vorgetragen wurden Überlegungen zur Aufstellung einer Luftwaffe und zur Notwendigkeit von Küstenschutzstreitkräften.

Amt

Jansen berichtet über ein Gespräch mit dem luxemburgischen Außenminister Bech anläßlich der Übergabe des Beglaubigungsschreibens. Themen waren der Schuman-Plan und die Unterbringung des Generalkonsulats. Hohen Kommissar McCloy

Im Mittelpunkt stehen der Exportpreis für Kohle, der West-OstHandel, der Verteidigungsbeitrag der Bundesrepublik und die finanzielle Unterstützung der Wissenschaften. Bittenfeld

Herwarth weist darauf hin, daß bei der Ernennung eines Generalkonsuls keine Zustimmung des Empfangslands erforderlich sei, wohl aber bei der Errichtung eines Generalkonsulats.

Kielmansegg faßt die zehnte Besprechung mit Vertretern der AHK auf dem Petersberg über einen Verteidigungsbeitrag der Bundesrepublik zusammen. Themen waren die Errichtung von obersten militärischen Bundesbehörden, eine taktische Luftwaffe und leichte Seestreitkräfte sowie die Kosten eines Verteidigungsbeitrags.

\section{Bundeskanzler Adenauer}

Krekeler berichtet über ein Gespräch mit dem Abteilungsleiter im amerikanischen Außenministerium, Byroade. Im Mittelpunkt standen die Ablösung des Besatzungsstatuts und das Gesetz zur völkerrechtlichen Beendigung des Kriegszustands mit Deutschland. 
76

08.05. Vortragender Legationsrat a.D. Roediger, Paris, an das Auswärtige Amt

Roediger erörtert die italienische Haltung zur Konferenz für die Organisation einer europäischen Armee in Paris.

Blankenhorn informiert über die Mitteilung des Politischen Beraters im amerikanischen Hochkommissariat, Reber, daß der Wortlaut eines Schreibens des Bundeskanzlers Adenauer an die AHK zur Übernahme von Besatzungskosten geändert werden solle.

Salat berichtet über die Vorbereitungen zu Verhandlungen über Kulturabkommen mit Frankreich, Italien und Spanien.

79 09.05. Aufzeichnung des Leiters der Delegation für die Ablösung des Besatzungsstatuts, Grewe

Grewe erörtert den Inhalt einer allgemeinen Instruktion für die Delegation der Bundesrepublik vor einer Aufnahme von Verhandlungen mit Vertretern der AHK über die Ablösung des Besatzungsstatuts. das Auswärtige Amt

Mohr informiert über die Reaktionen auf den Vorschlag des französischen Außenministers Schuman, einen inoffiziellen $\mathrm{Ge}$ dankenaustausch zwischen den Außenministern der Mitgliedstaaten des Europarats durchzuführen.

81

10.05. Aufzeichnung des Vortragenden Legationsrats Dittmann

Dittmann notiert die Mitteilung des Politischen Beraters im britischen Hochkommissariat, O'Neill, zum Charakter der Besprechungen mit Vertretern der AHK auf dem Petersberg über die Ablösung des Besatzungsstatuts. Außenminister Schuman

Adenauer bittet Schuman, den amerikanischen Hohen Kommissar McCloy zu veranlassen, mit der Bundesregierung über die Bildung der neuen Kohle- und Stahlgesellschaften zu verhandeln, bevor die Durchführungsverordnungen der AHK in Kraft treten. 
83 16.05. Aufzeichnung des Vortragenden Legationsrats von Kessel, Paris

Kessel informiert über ein Gespräch mit dem Kabinettschef des Generalsekretärs im französischen Außenministerium, Laloy. Themen waren die Vorkonferenz der stellvertretenden Außenminister der Vier Mächte, die Verhandlungen über eine europäische Armee, die Aufnahme der Türkei und Griechenlands in die NATO und die „Internationale Konferenz zur Lösung des deutschen Problems“.

84 16.05. Vortragender Legationsrat a. D. Roediger, Paris, an das Auswärtige Amt

Roediger bilanziert die Verhandlungen in den Ausschüssen der Konferenz für die Organisation einer europäischen Armee. Vorsitzenden der Alliierten Hohen Kommission, McCloy

Adenauer bittet um beschleunigte Rückgabe der Akten des Auswärtigen Amts aus der Zeit vor 1945. Dittmann

Dittmann hält das Ergebnis eines Gesprächs des Bundeskanzlers Adenauer mit dem amerikanischen Hohen Kommissar McCloy über die Ablösung des Besatzungsstatuts durch vertragliche Regelungen fest.

Kielmansegg faßt die elfte Besprechung mit Vertretern der AHK auf dem Petersberg über einen Verteidigungsbeitrag der Bundesrepublik zusammen. Themen waren die obersten Verteidigungsbehörden, die territoriale Organisation von Truppenkontingenten sowie die Abfassung eines Schlußberichts.

88 19.05. Gespräch des Bundeskanzlers Adenauer mit dem britischen Außenminister Morrison

Adenauer und Morrison erörtern die innenpolitische Lage der Bundesrepublik, Verteidigungsfragen und die Haltung Großbritanniens zur europäischen Einigung. an das Auswärtige Amt

Kamphoevener faßt Gespräche im türkischen Außenministerium zusammen. Themen waren die Erleichterung der Konsulartätigkeit, die bilateralen Wirtschaftsbeziehungen, das deutsche Eigentum in der Türkei und die Aufnahme diplomatischer Beziehungen. 
Blankenhorn resümiert ein Gespräch mit dem türkischen Gesandten Ayasli zur Sicherheitslage der Türkei.

91 25.05. Aufzeichnung des Referenten Haack

Haack gibt Mitteilungen des Mitarbeiters im amerikanischen Hochkommissariat, Koczak, wieder, wonach eine Rückgabe der Akten des Auswärtigen Amts aus der Zeit vor 1945 nicht vor Abschluß der amerikanisch-britisch-französischen Aktenpublikation erfolgen werde, an der auch Historiker aus der Bundesrepublik beteiligt werden sollten. französischen Hohen Kommissar François-Poncet

Adenauer und François-Poncet erörtern die Auswirkungen des Verbots der DPS auf die deutsch-französischen Beziehungen.

Grewe erörtert notwendige vertragliche Vereinbarungen zur Ablösung des Besatzungsstatuts. Amt

Hausenstein berichtet, daß der Gesandte der Republik China (Taiwan) in Paris, Tuan Mao-Lan, den Wunsch nach Aufnahme diplomatischer Beziehungen geäußert habe. Staatssekretär Hallstein

Roediger regt an, bei der Ressortbesprechung am 5. Juni 1951 die Position der Bundesrepublik zu offenen Fragen der Konferenz für die Organisation einer europäischen Armee festzulegen.

96 04.06. Aufzeichnung des Obersten a.D. Graf von Kielmansegg

Kielmansegg faßt die zwölfte Besprechung mit Vertretern der AHK auf dem Petersberg über einen Verteidigungsbeitrag der Bundesrepublik zusammen. Verabschiedet wurde der erste Teil des Zwischenberichts.

Kielmansegg faßt die dreizehnte Besprechung mit Vertretern der AHK auf dem Petersberg über einen Verteidigungsbeitrag der Bundesrepublik zusammen, in der der zweite Teil des Zwischenberichts verabschiedet wurde. 
98

05.06. Bundeskanzler Adenauer an den amerikanischen Hohen Kommissar McCloy

Adenauer übermittelt McCloy ein Memorandum über Maßnahmen zur Bekämpfung des illegalen West-Ost-Handels und stimmt dem amerikanischen Vorschlag einer Zusammenarbeit mit Sachverständigen aus der Bundesrepublik zu.

99

05.06. Ressortbesprechung

Nach Darlegungen über die Verhandlungen bei der Konferenz für die Organisation einer europäischen Armee in Paris erörtern die Teilnehmer der Ressortbesprechung den von französischer Seite gewünschten Zwischenbericht.

100 05.06. Instruktionen für die Delegation bei der Konferenz für die Organisation einer europäischen Armee in Paris

Das Vorgehen für die Abfassung eines Zwischenberichts wird festgelegt.

101 10.06. Vortragender Legationsrat a. D. Roediger, Paris, an Staatssekretär Hallstein

Roediger berichtet über ein Gespräch mit dem Leiter der französischen Delegation bei der Konferenz für die Organisation einer europäischen Armee in Paris, Alphand, in dem Zweck, Form und Inhalt eines Zwischenberichts erörtert wurden.

102 11.06. Aufzeichnung des Gesandtschaftsrats a.D. Weiz

Weiz faßt die Vorbesprechungen mit dem Drei-Mächte-Ausschuß für die deutschen Auslandsschulden vom 5. bis 7. Juni 1951 in Bonn und Bad Godesberg zusammen.

103

14.06. Aufzeichnung des Gesandten von Bargen

Bargen berichtet über die Aufnahme der Bundesrepublik in die Internationale Arbeitsorganisation.

104 15.06. Staatssekretär Hallstein an den Geschäftsführenden Vorsitzenden der Alliierten Hohen Kommission, François-Poncet

Hallstein übermittelt François-Poncet ein Memorandum zur Frage eines finanziellen Verteidigungsbeitrags. Hingewiesen wird auf die begrenzte Leistungsfähigkeit der Bundesrepublik aufgrund sozialer Verpflichtungen.

S. 306 
Hallstein berichtet von einem Gespräch mit dem amerikanischen Botschafter in Paris, Bruce, in dem Fragen der Konferenz für die Organisation einer europäischen Armee erörtert wurden.

107 20.06. Aufzeichnung des Vortragenden Legationsrats a. D. Roediger, Paris

Roediger informiert über ein Gespräch des Staatssekretärs Hallstein mit dem Leiter der französischen Delegation bei der Konferenz für die Organisation einer europäischen Armee in Paris, Alphand, zur Frage eines Verteidigungsbeitrags der Bundesrepublik.

108 20.06. Staatssekretär Hallstein, z. Z. Paris, an Bundeskanzler Adenauer

Hallstein faßt Gespräche mit dem amerikanischen Botschafter in Paris, Bruce, sowie mit dem Leiter der französischen Delegation bei der Konferenz für die Organisation einer europäischen Armee, Alphand, zusammen.

109 21.06. Runderlaß des Referenten Schaffarczyk

Schaffarczyk übermittelt die Weisung der AHK, Teilnehmern an den Internationalen Festspielen der Kommunistischen Jugend in Ost-Berlin keine Erlaubnis zur Ein- und Durchreise in die Bundesrepublik zu erteilen.

110 22.06. Staatssekretär Hallstein, z.Z. Paris, an Bundeskanzler Adenauer

Hallstein informiert über die Aufnahme der Bundesrepublik in die UNESCO und über ein Gespräch mit dem Generalkommissar des französischen Planungsamts, Monnet, zu den Verhandlungen über eine europäische Armee.

111 22.06. Aufzeichnung des Leiters der Delegation für die Ablösung des Besatzungsstatuts, Grewe

Grewe berichtet über ein Gespräch mit dem Politischen Berater im amerikanischen Hochkommissariat, Reber, zu Fragen der Ablösung des Besatzungsstatuts.

112 25.06. Vortragender Legationsrat von Kessel, Paris, an das Auswärtige Amt

Kessel berichtet über ein Gespräch mit dem Kabinettschef des Generalsekretärs im französischen Außenministerium, Laloy, nach Abbruch der Vorkonferenz der stellvertretenden Außenminister der Vier Mächte in Paris. 
113 28.06. Aufzeichnung des Leiters der Delegation für die Ablösung des Beatzungsstatuts, Grewe

Grewe nimmt zum Truppenvertrag Stellung und plädiert dafür, den Drei Mächten begrenzte Befugnisse zur Verhängung des Ausnahmezustands einzuräumen.

114

28.06. Bundeskanzler Adenauer an den Geschäftsführenden Vorsitzenden der Alliierten Hohen Kommission, François-Poncet

Adenauer weist darauf hin, daß der Bundestag die Anerkennung der deutschen Auslandsschulden überprüfen könnte, falls die Frage des deutschen Auslandsvermögens nicht geregelt werde.

115 29.06. Ressortbesprechung

In der Ressortbesprechung zu Fragen der Ablösung des Besatzungsstatuts wird insbesondere die Hinnahme von Ausnahmebefugnissen aufgrund der Obersten Gewalt der Drei Mächte erörtert.

116 30.06. Aufzeichnung des Leiters der Delegation für die Ablösung des Besatzungsstatuts, Grewe

Grewe faßt die Besprechungen mit Vertretern der AHK auf dem Petersberg über die Ablösung des Besatzungsstatuts zusammen.

117 02.07. Aufzeichnung des Botschaftsrats a.D. Becker

Becker informiert über israelische Vorschläge für den Warenverkehr mit der Bundesrepublik.

118 02.07. Generalkonsul I. Klasse Krekeler, Washington, an das Auswärtige Amt

Krekeler berichtet von einem Gespräch mit dem Abteilungsleiter im amerikanischen Außenministerium, Byroade, über eine völkerrechtliche Beendigung des Kriegszustandes mit Deutschland.

119 05.07. Gespräch des Bundeskanzlers Adenauer mit dem amerikanischen Hohen Kommissar McCloy

Adenauer und McCloy erörtern den West-Ost-Handel, die ECAHilfe, die Durchführung des Gesetzes Nr. 27 der AHK, den Verteidigungsbeitrag der Bundesrepublik und die Ablösung des Besatzungsstatuts. 

Ablösung des Besatzungsstatuts, Grewe

Grewe berichtet von Gesprächen mit dem Leiter der Abteilung für Politische Angelegenheiten im französischen Hochkommissariat, de Guiringaud, und dem Politischen Berater im britischen Hochkommissariat, O'Neill. Themen waren die Vorschläge der Drei Mächte zur Aufhebung der Kontrollen auf dem Gebiet der auswärtigen Beziehungen.

121 06.07. Bundeskanzler Adenauer an den Geschäftsführenden Vorsitzenden der Alliierten Hohen Kommission, Kirkpatrick

Adenauer übermittelt Kirkpatrick ein Memorandum zur Problematik verschleppter Personen und Flüchtlinge im Bundesgebiet.

Schmoller berichtet über ein Gespräch mit dem Politischen Berater im britischen Hochkommissariat, O'Neill. Erörtert wurden die Konsequenzen der Ablösung des Besatzungsstatuts für die Oberste Gewalt der Drei Mächte.

London, an das Auswärtige Amt

Abs teilt mit, daß er sich bei den Besprechungen über die deutschen Vorkriegsschulden in London lediglich vorbehalten habe, die Einbeziehung des deutschen Auslandsvermögens zu einem späteren Zeitpunkt vorzubringen. die Organisation einer europäischen Armee in Paris

Der Beauftragte des Bundeskanzlers, Blank, wird zum Leiter der Delegation bei der Konferenz für die Organisation einer europäischen Armee in Paris ernannt. Die Delegation erhält Weisungen für die weiteren Verhandlungen und für die Abfassung eines Zwischenberichts.

125 10.07. Sitzung des Lenkungsausschusses der Konferenz für die Organisation einer europäischen Armee in Paris

In der Sitzung des Lenkungsausschusses werden insbesondere die Rekrutierung von Soldaten in nationaler Verantwortlichkeit während einer Übergangsperiode und die Befugnisse eines europäischen Verteidigungskommissars erörtert. 
126 10.07. Aufzeichnung des Vortragenden Legationsrats

\section{Dittmann}

Dittmann berichtet über Verfahren gegen Deutsche, die wegen Kriegsverbrechen in den westlichen Staaten angeklagt oder verurteilt wurden. Er erörtert die jeweiligen Möglichkeiten zur Einflußnahme auf Begnadigungen.

127 11.07. Gespräch des Leiters der Delegation für die Ablösung des Besatzungsstatuts, Grewe, mit dem Berliner Senator für Bundesangelegenheiten, Klein

Thema ist die Einbeziehung von Berlin (West) in die Verhandlungen und in das Vertragswerk über die Ablösung des Besatzungsstatuts.

128 11.07. Aufzeichnung des Leiters der Delegation für die Ablösung des Besatzungsstatuts, Grewe

Grewe unterbreitet den Entwurf einer Grundsatzerklärung der Drei Mächte zur Ablösung des Besatzungsstatuts.

129 14.07. Aufzeichnung des Obersten a.D. Graf von Kielmansegg, Paris

Kielmansegg informiert über das amerikanische Drängen auf rasche Aufstellung von militärischen Einheiten der Bundesrepublik.

130 19.07. Sicherheitsvertrag (Entwurf)

In dem Entwurf eines Sicherheitsvertrags werden die Beziehungen zwischen der Bundesrepublik und den Drei Mächten neu geregelt.

131 19.07. Aufzeichnung des Leiters der Delegation für die Ablösung des Besatzungsstatuts, Grewe

Grewe informiert über Gespräche mit britischen und amerikanischen Vertretern der AHK zu Fragen der Ablösung des Besatzungsstatuts durch vertragliche Regelungen.

132 21.07. Aufzeichnung des Vortragenden Legationsrats a. D. Roediger und des Ministerialrats Ophüls, Paris

Roediger und Ophüls berichten über die amerikanische Haltung zur Schaffung einer europäischen Armee unter Beteiligung der Bundesrepublik. 
Böker faßt eine Unterredung mit dem ehemaligen Sicherheitsberater des Bundeskanzlers zusammen. Graf von Schwerin berichtete von einem Aufenthalt in Großbritannien und den Äußerungen seiner Gesprächspartner zu einem Verteidigungsbeitrag der Bundesrepublik.

134 24.07. Vortragender Legationsrat a. D. Roediger, Paris, an das Auswärtige Amt

Roediger teilt mit, daß der Zwischenbericht von der Konferenz für die Organisation einer europäischen Armee in Paris genehmigt und mit der Stellungnahme der Delegation der Bundesrepublik an die Beobachter- und Teilnehmerstaaten weitergeleitet worden sei.

135 25.07. Vortragender Legationsrat a. D. Roediger, Paris, an das Auswärtige Amt

Roediger berichtet, daß bei einem Gespräch mit dem britischen Gesandten in Paris, Hayter, vom Beauftragten des Bundeskanzlers, Blank, der Wunsch nach einer aktiveren Rolle des britischen Beobachters auf der Konferenz für die Organisation einer Europäischen Verteidigungsgemeinschaft geäußert wurde.

136 02.08. Sicherheitsvertrag (Entwurf)

In dem mit den Drei Mächten zu schließenden Vertrag sollen nach der völkerrechtlichen Beendigung des Kriegszustands die gegenseitigen Verpflichtungen zwischen der Bundesrepublik und den Drei Mächten neu geregelt werden.

137 09.08. Memorandum der Bundesregierung (Entwurf)

Die Bundesregierung schlägt vor, das Saargebiet zu einem europäischen Territorium zu erklären und den an der Europäischen Gemeinschaft für Kohle und Stahl beteiligten Staaten die oberste Regierungsgewalt zu übertragen.

138 10.08. Aufzeichnung des Vortragenden Legationsrats von Etzdorf

Etzdorf unterrichtet über ein Gespräch mit dem stellvertretenden Präsidenten des Polnischen Nationalrats in London, Makkiewicz, über die deutsch-polnische Grenzfrage.

Blankenhorn gibt Äußerungen des französischen Stellvertretenden Hohen Kommissars Bérard zu Reisen des ehemaligen Botschafters Rahn nach Spanien und Portugal wieder. 
Blankenhorn faßt ein Gespräch des Bundeskanzlers Adenauer mit dem amerikanischen Stellvertretenden Hohen Kommissar Hays über den Entwurf der Bundesregierung vom 2. August 1951 für einen Sicherheitsvertrag zusammen.

141 14.08. Aufzeichnung des Leiters der Delegation für die Ablösung des Besatzungsstatuts, Grewe

Grewe bilanziert die Besprechungen vom 10. Mai bis 3. August 1951 mit Vertretern der AHK auf dem Petersberg über die Ablösung des Besatzungsstatuts. Auswärtige Amt

Ulrich berichtet über seinen Antrittsbesuch bei Ministerpräsident Tito. Im Mittelpunkt standen die wirtschaftliche Zusammenarbeit und die Rückführung deutscher Kriegsgefangener aus Jugoslawien.

143 23.08. Bundeskanzler Adenauer an den französischen Außenminister Schuman

Adenauer unterbreitet Schuman Überlegungen zu einem Vertragswerk über die Ablösung des Besatzungsstatuts. Zugleich äußert er sich besorgt über die Belastung der bilateralen Beziehungen durch die Saarfrage und die von Frankreich wegen Kriegsverbrechen verurteilten Deutschen.

\section{25.08. Memorandum der Bundesregierung}

Entwickelt werden Überlegungen zu einer Vorvereinbarung über eine europäische Verteidigungsgemeinschaft, aufgrund derer gesetzliche und militärische Maßnahmen zur Schaffung europäischer Streitkräfte unmittelbar eingeleitet werden können.

145 25.08. Erklärung der Bundesregierung (Entwurf)

Die Bundesregierung nimmt Stellung zum Problem der Wiedergutmachung gegenüber Israel und den Juden.

Blankenhorn faßt ein Gespräch des Bundeskanzlers mit dem britischen Hohen Kommissar zusammen. Adenauer und Kirkpatrick erörterten den Entwurf der Bundesregierung für einen Sicherheitsvertrag, das geplante Ablösungsgesetz zum Kontrollratsgesetz Nr. 5 über das deutsche Auslandsvermögen sowie die beschleunigte Freilassung von Deutschen, die wegen Kriegsverbrechen von britischen Militärgerichten verurteilt wurden. 
Hess erörtert einen Austausch von amtlichen Vertretungen zwischen der Bundesrepublik und ostasiatischen bzw. südostasiatischen Staaten.

Themen waren die innenpolitische Lage der Bundesrepublik, die Ablösung des Besatzungsstatuts, ein Verteidigungsbeitrag der Bundesrepublik sowie Probleme der europäischen Kohleund Stahlindustrie.

\section{04.09. Aufzeichnung des Vortragenden Legationsrats von} Kessel, Paris

Kessel berichtet von einem Gespräch des Beauftragten des Bundeskanzlers, Blank, mit dem Generalkommissar des französischen Planungsamts, Monnet, und dem Leiter der französischen Delegation bei der Konferenz für die Organisation einer Europäischen Verteidigungsgemeinschaft, Alphand. Themen waren die Freistellung von französischen Truppen für Übersee und die Teilnahme des Generalleutnants a. D. Speidel an den Verhandlungen über eine Europäische Verteidigungsgemeinschaft.

\section{10.09. Aufzeichnung des Staatssekretärs Hallstein}

Hallstein faßt ein Gespräch mit dem Abteilungsleiter in der amerikanischen Hohen Kommission, Leonard, über Kürzungen der amerikanischen Wirtschaftshilfe zusammen.

\section{Dittmann}

Dittmann bittet einzelne Amtsangehörige um dienstliche Stellungnahmen zu Vorwürfen, die in einer Artikelserie der „Frankfurter Rundschau" erhoben wurden. Amt

Du Mont berichtet über die Haltung der niederländischen Regierung zu einer europäischen Verteidigungsgemeinschaft.

\section{14.09. Aufzeichnung des Referenten Böker}

Böker informiert über eine Unterredung mit dem französischen Stellvertretenden Hohen Kommissar. Bérard berichtete über die Ergebnisse der Außenministerkonferenz der Drei Mächte in Washington. 
Im Mittelpunkt der Besprechung stehen eine weitgehende Angleichung der Rechtsstellung von Berlin (West) an diejenige der Bundesrepublik nach Ablösung des Besatzungsstatuts und die Ausübung der Kontrollrechte durch die Drei Mächte in der Stadt. Vorsitzenden der Alliierten Hohen Kommission, François-Poncet

Adenauer informiert über den Abschluß des Interzonenhandelsabkommens am 20. September 1951 und über die Bedingungen, die von der Bundesregierung gegenüber der DDR gestellt wurden.

Etzdorf übermittelt eine Sprachregelung für die Bezeichnung der Gebiete des Deutschen Reiches östlich von Oder und Neiße. Kommission

Staatssekretär Hallstein erörtert mit Vertretern der AHK die Ausführungen des französischen Hohen Kommissars FrançoisPoncet über die Außenministerkonferenz der Drei Mächte in Washington.

Unter Vorsitz des Staatssekretärs Hallstein wird die Position der Bundesregierung zu den einzelnen Punkten erörtert, die mit den Drei Mächten bei der Ablösung des Besatzungsstatuts vertraglich zu regeln sind.

Staatssekretär Hallstein nimmt Stellung zum Entwurf der Drei Mächte vom 24. September 1951 für einen Generalvertrag.

Böker faßt ein Gespräch mit dem Vertreter des American Jewish Committee in Europa zusammen, in dem Shuster am 26. September 1951 vorab über die Regierungserklärung zur Wiedergutmachungsfrage informiert wurde. 
161
03.10. Bundeskanzler Adenauer an den Geschäftsführenden Vorsitzenden der Alliierten Hohen Kommission, Kirkpatrick

Adenauer bittet die Drei Mächte, die Bildung einer internationalen Kommission unter der Kontrolle der UN vorzuschlagen, welche die Voraussetzungen für freie gesamtdeutsche Wahlen prüfen soll.

162

04.10. Aufzeichnung des Staatssekretärs Hallstein

Hallstein lehnt die Errichtung eines Europaministeriums ab.

163

12.10. Aufzeichnung des Referenten Grosse, Washington

Grosse berichtet von einer informellen Besprechung mit Vertretern des Internationalen Währungsfonds und der Weltbank über einen Beitritt der Bundesrepublik.

164 12.10. Besprechung mit Vertretern der Alliierten Hohen Kommission

Der zur Vorbereitung der Verträge zwischen den Drei Mächten und der Bundesrepublik eingesetzte Hauptausschuß befaßt sich mit den Vorbehaltsrechten der Drei Mächte.

165 13.10. Ressortbesprechung

Thema ist die Organisation der europäischen Verteidigung.

166 15.10. Generalkonsul I. Klasse Krekeler, Washington, an das Auswärtige Amt

Krekeler informiert über eine Unterredung mit dem Berater im amerikanischen Außenministerium, Bohlen. Erörtert wurden die sowjetische Haltung zur Wiedervereinigung Deutschlands und Fragen der Integration der Bundesrepublik in das westliche Staatensystem.

167

17.10. Gesandter Sieveking, Stockholm, an das Auswärtige Amt

Sieveking berichtet über die schwedische Position zur Eingliederung der Bundesrepublik in das westliche Staatensystem und zur Wiedervereinigung Deutschlands.

168

18.10. Besprechung mit Vertretern der Alliierten Hohen Kommission

Im Mittelpunkt stehen die Vorbehaltsrechte der Drei Mächte in bezug auf Berlin, die Stationierung von Truppen im Bundesgebiet und gesamtdeutsche Fragen.

S. 518 
In der Ressortbesprechung steht die Zuständigkeitsabgrenzung zwischen Kommissar, Rat und Versammlung der Europäischen Verteidigungsgemeinschaft im Mittelpunkt. Kommission

Erörtert werden Fragen im Zusammenhang mit den Vorbehaltsrechten der Drei Mächte für Berlin und mit dem Schutz der alliierten Truppen in der Bundesrepublik. Kommissaren François-Poncet, Kirkpatrick und McCloy

Behandelt werden der Berlin-Verkehr und das Interzonenhandelsabkommen vom 20. September 1951. der europäischen Sektion des World Jewish Congress, Barou

Blankenhorn regt vertrauliche Vorgespräche an, um möglichst bald Verhandlungen über Umfang und Modalitäten von Wiedergutmachungsleistungen der Bundesrepublik aufnehmen zu können. Kommission

In der ersten Besprechung des Unterausschusses für den Truppenvertrag wird der Vertragsentwurf der Drei Mächte erörtert.

Erläutert werden einzelne Artikel für einen Generalvertrag und dabei die Fassung der Bundesregierung und diejenige der Drei Mächte gegenübergestellt.

Salat regt an, Planstellen für Kulturreferenten an den Auslandsvertretungen zu schaffen.

Im Hinblick auf die Europäische Verteidigungsgemeinschaft werden die Kompetenzen des Ministerrats gegenüber dem Verteidigungskommissar, die Organisation des Verteidigungskommissariats, Abstimmungsmodalitäten im Ministerrat und der Verwaltungsaufbau erörtert. 
177 05.11. Gespräch des Bundeskanzlers Adenauer mit dem italienischen Botschafter Babuscio Rizzo

Themen sind die Zusage des Ministerpräsidenten de Gasperi zu einem Besuch in der Bundesrepublik, die Einsetzung einer UNKommission zur Prüfung der Voraussetzungen für freie gesamtdeutsche Wahlen sowie die Verhandlungen mit der AHK über die Ablösung des Besatzungsstatuts.

$178 \quad 06.11$. Ressortbesprechung

Auf Bitte der UNESCO wird beschlossen, zwei Delegierte zu einer Besprechung über die Errichtung eines europäischen Laboratoriums für Kernphysik zu entsenden.

179 07.11. Ministerialdirektor Blankenhorn, z.Z. Paris, an Bundeskanzler Adenauer

Blankenhorn informiert über ein Gespräch mit dem ehemaligen Kabinettsdirektor des französischen Außenministers, Clappier, zur französischen Europa- und Deutschlandpolitik sowie zum Beginn der Sitzungsperiode der UN-Generalversammlung in Paris.

180 07.11. Aufzeichnung des Gesandten a.D. Kroll, Bundesministerium für Wirtschaft

S. 601

Kroll faßt ein Gespräch des Bundeskanzlers Adenauer und des Bundesministers Erhard mit dem amerikanischen Hohen Kommissar McCloy über den Berlin-Verkehr und das Interzonenhandelsabkommen vom 20. September 1951 zusammen.

181 08.11. Aufzeichnung des Ministerialdirektors Blankenhorn, z.Z. Paris

Blankenhorn informiert über eine Unterredung mit dem Generalkommissar des französischen Planungsamts, Monnet. Im Mittelpunkt stand die französische Europa- und Deutschlandpolitik.

Hallstein legt Aufgabenbereich und Modalitäten der Berichterstattung der Auslandsvertretungen fest.

183 12.11. Staatssekretär Hallstein an die Delegation bei der Konferenz für die Organisation einer Europäischen Verteidigungsgemeinschaft in Paris

Hallstein erteilt Weisungen zur Organisation des Kommissariats, zu den Befugnissen des Ministerrats sowie zur territorialen Organisation einer Europäischen Verteidigungsgemeinschaft. 
Blankenhorn berichtet über Gespräche mit dem Generalkommissar des französischen Planungsamts, Monnet, und mit dem Kabinettsdirektor des französischen Außenministers, BourbonBousset. Hauptthemen waren die Europäische Verteidigungsgemeinschaft und der bevorstehende Besuch des Bundeskanzlers Adenauer in Paris.

Speidel erläutert dem französischen Vertreter im Ausschuß der militärischen Chefdelegierten der Konferenz für die Organisation einer Europäischen Verteidigungsgemeinschaft, General de Larminat, Vorschläge zur Aufstellung von Streitkräften und zur territorialen Organisation einer Europäischen Verteidigungsgemeinschaft. Auswärtige Amt

Blankenhorn berichtet, daß die UN-Generalversammlung die Einsetzung einer Kommission auf ihre Tagesordnung gesetzt habe, welche die Voraussetzungen für freie gesamtdeutsche Wahlen prüfen soll. Bundeskanzler Adenauer

Blankenhorn informiert über ein Gespräch mit dem Abteilungsleiter im amerikanischen Außenministerium, Byroade. Thema waren die verschiedenen Positionen zur Wiedervereinigung Deutschlands unter Einbeziehung der Gebiete östlich der OderNeiße-Linie.

Erörtert werden die vom belgischen Außenminister van Zeeland vorgetragenen Bedenken gegen den supranationalen Charakter einer Europäischen Verteidigungsgemeinschaft. Ablösung des Besatzungsstatuts, Grewe

Grewe analysiert die Positionen der Vier Mächte zur Wiedervereinigung Deutschlands in den Grenzen von 1937. britischen Außenminister Eden in Paris

Im Mittelpunkt stehen die UN-Kommission zur Prüfung der Voraussetzungen für freie gesamtdeutsche Wahlen, die $\mathrm{Be}$ schränkung der Rüstungsproduktion und der Verteidigungsbeitrag der Bundesrepublik. 
191 22.11. Gespräch des Bundeskanzlers Adenauer mit dem

S. 630 französischen Verteidigungsminister Bidault in Paris

Hauptthemen sind die Verhandlungen über die Ablösung des Besatzungsstatuts und der geplante Besuch des Generals de Gaulle in Aachen.

192 22.11. Gespräch des Bundeskanzlers Adenauer mit den Außenministern Acheson, Eden und Schuman in Paris

Im Anschluß an Ausführungen des Bundeskanzlers Adenauer zur innenpolitischen Lage der Bundesrepublik billigen die AuBenminister den Entwurf des Generalvertrags zwischen der Bundesrepublik und den Drei Mächten.

193 22.11. Generalvertrag (Entwurf)

Die Beziehungen zwischen der Bundesrepublik und den Drei Mächten werden neu geregelt.

Blankenhorn faßt ein Gespräch des Bundeskanzlers Adenauer mit dem amerikanischen Hohen Kommissar McCloy über die NATO-Ministerratstagung in Rom und die innenpolitische Lage in Frankreich zusammen. Auswärtige Amt

Brentano berichtet von einem Gespräch mit dem Unterstaatssekretär im italienischen Außenministerium, Taviani, über die NATO-Ministerratstagung in Rom.

Im Mittelpunkt stehen Probleme der europäischen Einigung, die Politik gegenüber der UdSSR, die Freilassung der wegen Kriegsverbrechen von britischen Militärgerichten verurteilten Deutschen sowie die innenpolitische Lage der Bundesrepublik.

197 04.12. Botschafter Oellers, Rio de Janeiro, an das Auswärtige Amt

Oellers unterbreitet Vorschläge zur Intensivierung der Zusammenarbeit zwischen den Auslandsvertretungen der Bundesrepublik in den lateinamerikanischen Staaten.

198 05.12. Gespräch des Leiters der Delegation für Auslandsschulden, Abs, mit den Delegationsleitern Gregh, Pierson und Rendel in London

Im Mittelpunkt steht die Regelung der deutschen Nachkriegsschulden. 
199

05.12. Referent Böker, z.Z. Paris, an das Auswärtige Amt

Böker informiert über den Beschluß des Politischen ad hoc-Ausschusses der UN-Generalversammlung, Vertreter der Bundesrepublik und von Berlin (West) sowie der DDR und von OstBerlin über die Einsetzung einer UN-Kommission anzuhören, welche die Voraussetzungen für freie gesamtdeutsche Wahlen prüfen soll.

\subsection{Hauserlaß des Staatssekretärs Hallstein}

Hallstein weist die Abteilungsleiter des Auswärtigen Amts an, die Auslandsvertretungen regelmäßig über die Politik der Bundesregierung mittels Informationserlassen zu unterrichten.

Blankenhorn erläutert dem schwedischen Gesandten Kumlin Einwände gegen den Vorschlag, die Vier Mächte über die Voraussetzungen und Bedingungen für die Durchführung freier gesamtdeutscher Wahlen beraten zu lassen.

14.12. Aufzeichnung des Generalleutnants a.D. Speidel, Paris

S. 668

Speidel hält eine Erklärung zur territorialen Organisation einer Europäischen Verteidigungsgemeinschaft fest, die er gegenüber General de Larminat vor der Sitzung des Ausschusses der militärischen Chefdelegierten der Konferenz für die Organisation einer Europäischen Verteidigungsgemeinschaft abgab.

14.12. Ministerialdirektor Blankenhorn an Generalkonsul I. Klasse Krekeler, Washington

Blankenhorn informiert über die Ergebnisse der Unterredung des Bundeskanzlers Adenauer mit dem Vorsitzenden des World Jewish Congress, Goldmann, am 6. Dezember 1951 in London zur Wiedergutmachung.

Hallstein faßt die Ergebnisse der Außenministerkonferenz in Straßburg über eine Europäische Verteidigungsgemeinschaft zusammen. 

Kommission

Im Mittelpunkt stehen die Verhandlungen über die Einsetzung eines Schiedsgerichts, über die Entflechtung der Montan- sowie der chemischen Industrie, über das geplante Kartellgesetz und über den Truppenvertrag. Blankenhorn

Böker berichtet von einem Gespräch mit dem amerikanischen Vertreter im Politischen ad hoc-Ausschuß der UN-Generalversammlung, Cooper, über die Einsetzung einer Kommission, welche die Voraussetzungen für freie gesamtdeutsche Wahlen prüfen soll. amerikanischen Hohen Kommissar McCloy

Erörtert werden ein finanzieller Verteidigungsbeitrag der Bundesrepublik, eine mögliche Auflösung der Dienstgruppen, die Einsetzung einer Gnadenkommission für die wegen Kriegsverbrechen von alliierten Militärgerichten verurteilten Deutschen sowie Beschränkungen der Rüstungsproduktion in der Bundesrepublik.

\section{19.12. Rundschreiben des Staatssekretärs Hallstein}

Hallstein faßt für das Kabinett die Verhandlungen mit den Drei Mächten über die Zusatzabkommen zum Generalvertrag zusammen. Auslandsschulden, Abs

Abs resümiert die Besprechungen mit den Drei Mächten vom 26. November bis 10. Dezember 1951 in London über die deutschen Nachkriegsschulden.

Krekeler berichtet von Ausführungen des Abteilungsleiters im amerikanischen Außenministerium, Byroade, über die UN-Kommission, welche die Voraussetzungen für freie gesamtdeutsche Wahlen prüfen soll.

Bundesminister Schäffer erörtert mit Vertretern der AHK die Höhe und das Berechnungsverfahren für einen finanziellen Verteidigungsbeitrag der Bundesrepublik. 
213 23.12. Professor Heisenberg an Staatssekretär Hallstein

Heisenberg berichtet über die UNESCO-Konferenz in Paris. Thema war die Errichtung eines europäischen Laboratoriums für Kernphysik.

214 27.12. Außenministerkonferenz in Paris

Die Außenminister beraten über Übergangsbestimmungen und Kompetenzen der einzelnen Organe einer Europäischen Verteidigungsgemeinschaft.

215 28.12. Ministerkonferenz in Paris

Die Außen- und Finanzminister erörtern haushaltsrechtliche Fragen einer Europäischen Verteidigungsgemeinschaft.

216 30.12. Ministerkonferenz in Paris

S. 735

Die Ministerkonferenz über eine Europäische Verteidigungsgemeinschaft befaßt sich mit dem Bericht der Finanzminister über Budget-Fragen und mit dem Schlußkommuniqué. 


\section{Literaturverzeichnis}

AAPD 1949/50

AAPD, Hohe Kommissare 1949-1951

AAPD, Hohe Kommissare 1952

ABS, Entscheidungen

ADENAUER, Briefe 1949-1951

ADENAUER, Briefe 1951-1953

ADENAUER, Erinnerungen 1945-1953

ADENAUER, Erinnerungen 1953-1955

ADENAUER, Teegespräche 1950-1954

ADENAUER ZUR POLITISCHEN LAGE 1946-1949

AdG 1951

AMTSBLATT DER AHK
Akten zur Auswärtigen Politik der Bundesrepublik Deutschland, hrsg. im Auftrag des Auswärtigen Amts vom Institut für Zeitgeschichte. Jahresband 1949/50, München 1997.

Akten zur Auswärtigen Politik der Bundesrepublik Deutschland. Band 1: Adenauer und die Hohen Kommissare 1949-1951, hrsg. von HansPeter Schwarz in Verbindung mit Reiner Pommerin, München 1989.

Akten zur Auswärtigen Politik der Bundesrepublik Deutschland. Band 2: Adenauer und die Hohen Kommissare 1952, hrsg. von HansPeter Schwarz in Verbindung mit Reiner Pommerin, München 1990.

Hermann J. Abs, Entscheidungen 1949-1953. Die Entstehung des Londoner Schuldenabkommens, 2. Auflage, Mainz 1991.

Adenauer. Rhöndorfer Ausgabe: Briefe 19491951, hrsg. von Rudolf Morsey und Hans-Peter Schwarz, bearbeitet von Hans Peter Mensing, Berlin 1985.

Adenauer. Rhöndorfer Ausgabe: Briefe 19511953, hrsg. von Rudolf Morsey und Hans-Peter Schwarz, bearbeitet von Hans Peter Mensing, Berlin 1987.

Konrad Adenauer, Erinnerungen 1945-1953, Stuttgart 1965.

Konrad Adenauer, Erinnerungen 1953-1955, Stuttgart 1966.

Adenauer. Rhöndorfer Ausgabe: Teegespräche 1950-1954, hrsg. von Rudolf Morsey und HansPeter Schwarz, bearbeitet von Hanns Jürgen Küsters, Berlin 1984.

Hanns Jürgen Küsters und Hans Peter Mensing, Konrad Adenauer zur politischen Lage 1946-1949. Aus den Berichten des schweizerischen Generalkonsuls in Köln Franz Rudolf v. Weiss. Dokumentation, in: Vierteljahrshefte für Zeitgeschichte 32 (1984), S. 289-317.

Keesing's Archiv der Gegenwart. Jahrgang 21, 1951 , zusammengestellt von Heinrich von Siegler, Wien [o. J.].

Amtsblatt der Alliierten Hohen Kommission in Deutschland. Nr. 1-72 [o.O.] 1949-1951. 


\author{
AMTSBLATT DER MILITÄRREGIERUNG \\ DEUTSCHLAND (Amerikanisches \\ Kontrollgebiet)
AMTSBLATT DER MILITÄRREGIERUNG DEUTSCHLAND (Britisches Kontroll- gebiet)

AMTSBLATT DES FRANZÖSISCHEN OBERKOMMANDOS IN DEUTSCHLAND

AMTSBLATT DES KONTROLLRATS

AMTSBlatT DES SAARLANDES

L’ANNÉE POLITIQUE 1951

AUSSENPOLITIK DER DDR I

AUSWÄRTIGER AUSSCHUSS

BDFD I

BDFD II

BEENDIGUNG DES KRIEGSZUSTANDS
Amtsblatt der Militärregierung Deutschland. Amerikanisches Kontrollgebiet. Ausgabe A-O, hrsg. vom Office of Military Government for Germany (U.S.), [Frankfurt/Main] 1946-1949.

Amtsblatt der Militärregierung Deutschland. Nr. 1-3: Kontrollgebiet der 21. Armeegruppe; Nr. 4-28: Britisches Kontrollgebiet; Nr. 29-36: Amtsblatt der Kontrollkommission - Deutschland (Britische Zone), [1945-1949].

Amtsblatt des französischen Oberkommandos in Deutschland. Nr. 1-305, [1945-1949].

Amtsblatt des Kontrollrats in Deutschland, hrsg. vom Alliierten Sekretariat. Nr. 1-19, Berlin 1945-1948; Ergänzungsblatt Nr. 1, Berlin 1946.

Amtsblatt des Saarlandes 1947-1951, hrsg. vom Informationsamt der Regierung des Saarlandes, Saarbrücken 1947-1951.

L'Année Politique 1951. Revue chronologique des principaux faits politiques, économiques et sociaux de la France et de l'Union française du Ier Janvier 1951 au 1er Janvier 1952, Paris [1952].

Dokumente zur Außenpolitik der Regierung der Deutschen Demokratischen Republik. Band I: Von der Gründung der Deutschen Demokratischen Republik am 7. Oktober 1949 bis zur Souveränitätserklärung am 25. März 1954, hrsg. vom Deutschen Institut für Zeitgeschichte, Berlin [Ost] 1954.

Der Auswärtige Ausschuß des Deutschen Bundestages. Sitzungsprotokolle 1949-1953. 1. Halbband: Oktober 1949 bis Mai 1952, bearbeitet von Wolfgang Hölscher, Düsseldorf 1998.

Die Bundesrepublik Deutschland und Frankreich. Dokumente 1949 bis 1963. Band 1: AuBenpolitik und Diplomatie, hrsg. von Horst Möller und Klaus Hildebrand, bearbeitet von Ulrich Lappenküper, München 1997.

Die Bundesrepublik Deutschland und Frankreich: Dokumente 1949 bis 1963. Band 2: Wirtschaft, hrsg. von Horst Möller und Klaus Hildebrand, bearbeitet von Andreas Wilkens, München 1997.

Die Beendigung des Kriegszustands mit Deutschland nach dem Zweiten Weltkrieg, bearbeitet von Hermann Mosler und Karl Doehring, Köln/Berlin 1963. 
BEMÜHUNGEN

BÉRARD, Ambassadeur

BERLIN 1945-1951

BERLIN 1951-1954

BLANKENHORN, Verständnis

BR DRUCKSACHEN

BR SITZUNGSBERICHTE

BT ANLAGEN

BT STENOGRAPHISCHE BERICHTE

BUNDESANZEIGER

BUNDESARBEITSBLATT 1951

BUNDESGESETZBLATT

CDU-BUNDESVORSTAND
Die Bemühungen der Bundesrepublik um Wiederherstellung der Einheit Deutschlands durch gesamtdeutsche Wahlen. Dokumente und Akten. I. Teil: Oktober 1949-Oktober 1953, hrsg. vom Bundesministerium für gesamtdeutsche Fragen, 4. erweiterte Auflage, Bonn 1958.

Armand Bérard, Un Ambassadeur se souvient. Band 2: Washington et Bonn 1945-1955, Paris 1978.

Berlin. Quellen und Dokumente 1945-1951, hrsg. im Auftrage des Senats von Berlin, bearbeitet durch Hans J. Reichhardt, Hanns U. Treutler und Albrecht Lampe, 2 Halbbände, Berlin 1964.

Berlin. Chronik der Jahre 1951-1954, hrsg. im Auftrage des Senats von Berlin, bearbeitet durch Hans J. Reichhardt, Joachim Drogmann und Hanns U. Treutler, Berlin 1968.

Herbert Blankenhorn, Verständnis und Verständigung. Blätter eines politischen Tagebuchs 1949 bis 1979, Frankfurt am Main/Berlin/Wien 1980.

Deutscher Bundesrat. Drucksachen, Bonn $1949 \mathrm{ff}$.

Deutscher Bundesrat. Sitzungsberichte, Bonn $1949 \mathrm{ff}$.

Verhandlungen des Deutschen Bundestages. Anlagen zu den Stenographischen Berichten, Bonn $1949 \mathrm{ff}$.

Verhandlungen des Deutschen Bundestages. Stenographische Berichte, Bonn 1949 ff.

Bundesanzeiger (bis August 1950: Bundesanzeiger und Öffentlicher Anzeiger für das Vereinigte Wirtschaftsgebiet. Bekanntmachungsorgan für das französische Besatzungsgebiet. Verkündungsorgan für die Verlautbarungen des Einfuhrausschusses), hrsg. vom Bundesminister der Justiz, Bonn 1949 ff.

Bundesarbeitsblatt, hrsg. vom Bundesminister für Arbeit. Jahrgang 1951, Stuttgart 1951.

Bundesgesetzblatt, hrsg. vom Bundesminister der Justiz, Köln 1949 ff.

Adenauer: „Es mußte alles neu gemacht werden." Die Protokolle des CDU-Bundesvorstandes 1950-1953, bearbeitet von Günter Buchstab, Stuttgart 1986. 


\section{CDU/CSU.FRAKTION}

CHARTER OF THE UNITED NATIONS

DBPO II

DEPARTMENT OF STATE BULLETIN

DEUTSCHE AUSLANDSSCHULDEN

DEUTSCHE AUSLANDSSCHULDEN, ENGLISCHES SONDERHEFT

DEUTSCHE AUSLANDSSCHULDEN, FRANZÖSISCHES SONDERHEFT

DEUTSCHES Vermögen IM AUSLAND

DEUTSCH-ISRAELISCHER DIALOG
Die CDU/CSU-Fraktion im Deutschen Bundestag 1949-1966. 1. Halbband: Sitzungsprotokolle 1949-1953, bearbeitet von Helge Heidemeyer, Düsseldorf 1998.

Charter of the United Nations. Commentary and Documents, hrsg. von Leland M. Goodrich, Edvard Hambro und Anne Patricia Simons, 3. überarbeitete Auflage, New York/London 1969.

Documents on British Policy Overseas. Series II. Band 1: The Schumann Plan, the Council of Europe and Western European Integration, May 1950-December 1952; Band 3: German Rearmament, September-December 1950, hrsg. von Roger Bullen und M. E. Pelly unter Mitarbeit von H. J. Yasamee und G. Bennett, London 1986-1989.

The Department of State Bulletin. The Official Weekly Record of United States Foreign Policy, Washington D.C. $1939 \mathrm{ff}$.

Deutsche Auslandsschulden. Dokumente zu den internationalen Verhandlungen, Oktober 1950 bis Juli 1951, hrsg. vom Auswärtigen Amt, dem Bundesministerium für Wirtschaft und dem Bundesministerium für den Marshallplan, Hameln [1951].

Deutsche Auslandsschulden. Dokumente zu den internationalen Verhandlungen, Oktober 1950 bis Juli 1951. Englisches Sonderheft, hrsg. vom Auswärtigen Amt, dem Bundesministerium für Wirtschaft und dem Bundesministerium für den Marshallplan, Hameln [1951].

Deutsche Auslandsschulden. Dokumente zu den internationalen Verhandlungen, Oktober 1950 bis Juli 1951. Französisches Sonderheft, hrsg. vom Auswärtigen Amt, dem Bundesministerium für Wirtschaft und dem Bundesministerium für den Marshallplan, Hameln [1951].

Deutsches Vermögen im Ausland. Internationale Vereinbarungen und ausländische Gesetzgebung. Mit Unterstützung des Bundesministeriums der Finanzen, des Bundesministeriums für Wirtschaft, des Bundesministeriums für den Marshallplan und der Bank deutscher Länder hrsg. vom Bundesministerium der Justiz, bearbeitet von Otto Böhmer, Konrad Duden und Hermann Jansen, Köln 1951.

Der deutsch-israelische Dialog. Dokumentation eines erregenden Kapitels deutscher Außenpolitik, hrsg. von Rolf Vogel, Teil I: Politik. Band 1, München/New York/London/Paris 1987. 
Literaturverzeichnis

\section{DOKUMENTE DES GETEILTEN DEUTSCHLAND}

DOKUMENTE ZUR BERLIN-FRAGE

DzD II/1

DzD II/2

DzD II/3

EUROPA-ARCHIV

EVG UND POLITISCHE FÖDERATION

FRUS 1943

FRUS 1948

FRUS 1949
Dokumente des geteilten Deutschland. Quellentexte zur Rechtslage des Deutschen Reiches, der Bundesrepublik Deutschland und der Deutschen Demokratischen Republik. Band 1, hrsg. von Ingo von Münch, 2. Auflage, Stuttgart 1976.

Dokumente zur Berlin-Frage 1944-1966, hrsg. vom Forschungsinstitut der Deutschen Gesellschaft für Auswärtige Politik e.V., Bonn in Zusammenarbeit mit dem Senat von Berlin, 3. durchgesehene und erweiterte Auflage, München 1967.

Dokumente zur Deutschlandpolitik. II. Reihe: Vom 9. Mai 1945 bis 4. Mai 1955. Band 1: Die Konferenz von Potsdam, 3 Teilbände, hrsg. vom Bundesminister des Innern, bearbeitet von Gisela Biewer, Neuwied/Frankfurt am Main 1992.

Dokumente zur Deutschlandpolitik. II. Reihe: Vom 9. Mai 1945 bis 4. Mai 1955. Band 2: Die Konstituierung der Bundesrepublik Deutschland und der Deutschen Demokratischen Republik 7. September bis 31. Dezember 1949, hrsg. vom Bundesministerium des Innern unter Mitwirkung des Bundesarchivs, bearbeitet von Hanns Jürgen Küsters unter Mitarbeit von Daniel Hoffmann, München 1996.

Dokumente zur Deutschlandpolitik. II. Reihe: Vom 9. Mai 1945 bis 4. Mai 1955. Band 3: 1. Januar bis 31. Dezember 1950, hrsg. vom Bundesministerium des Innern unter Mitwirkung des Bundesarchivs, bearbeitet von Hanns Jürgen Küsters, Daniel Hoffmann und Carsten Tessmer, München 1997.

Europa-Archiv. Zeitschrift für Internationale Politik, Frankfurt am Main 1946 ff.

Walter Lipgens, EVG und Politische Föderation. Protokolle der Konferenz der an den Verhandlungen über eine europäische Verteidigungsgemeinschaft beteiligten Länder am 11. Dezember 1951. Dokumentation, in: Vierteljahrshefte für Zeitgeschichte 32 (1984), S. 637-688.

Foreign Relations of the United States. Diplomatic Papers, 1943. Band I: General, Washington D.C. 1963.

Foreign Relations of the United States, 1948. Band II: Germany and Austria, Washington D.C. 1973.

Foreign Relations of the United States, 1949. Band III: Council of Foreign Ministers; Germany and Austria, Washington D.C. 1974. 
FRUS 1950

FRUS 1951

FRUS, The Conference at Malta and Yalta 1945

GERMANY 1947-1949

GESETZBLATT DER DDR

GESETZBLATT DER VERWALTUNG DES VEREINIGTEN WIRTSCHAFTSGEBIETES 1947-49

GOLDMANN, Staatsmann

GREWE, Rückblenden

HANSARD

HEUSS-ADENAUER, Briefwechsel

JOURNAL OFFICIEL, ASSEMBLÉE NATIONALE

JOURNAL OFFICIEL, CONSEIL DE LA RÉPUBLIQUE 1951
Foreign Relations of the United States, 1950. Band III: Western Europe, Washington D.C. 1977; Band IV: Central and Eastern Europe; The Soviet Union, Washington D. C. 1980.

Foreign Relations of the United States, 1951. Band I: National Security Affairs; Foreign Economic Policy; Band III: European Security and the German Question, 2 Teilbände; Band IV: Europe: Political and Economic Developments, 2 Teilbände; Band VI: Asia and the Pacific, Washington D.C. 1977-1985.

Foreign Relations of the United States. Diplomatic Papers. The Conference at Malta and Yalta 1945, Washington D.C. 1955.

Germany 1947-1949. The Story in Documents, hrsg. vom Department of State, Washington D.C. 1950 .

Gesetzblatt der Deutschen Demokratischen Republik, Berlin [Ost] 1949 ff.

Gesetzblatt der Verwaltung des Vereinigten Wirtschaftsgebietes 1947-49, hrsg. vom Büro des Wirtschaftsrates, Frankfurt am Main [o.J.].

Nahum Goldmann, Staatsmann ohne Staat. Autobiographie, Köln/Berlin 1970.

Wilhelm G. Grewe, Rückblenden 1976-1951, Frankfurt am Main/Berlin/Wien 1979.

Parliamentary Debates (Hansard). House of Commons, Official Report. Fifth Series. Bände 443-455 (Session 1947-48), London $1947 \mathrm{ff}$; Bände 480-492 (Session 1950-1951), London 1950/51.

Adenauer. Rhöndorfer Ausgabe: Heuss-Adenauer. Unserem Vaterland zugute. Der Briefwechsel 1948-1963, hrsg. von Rudolf Morsey und Hans-Peter Schwarz, bearbeitet von Hans Peter Mensing, Berlin 1989.

Journal Officiel de la République Française. Débats Parlementaires. Assemblée Nationale. Compte rendue in extenso des séances. Questions écrites et réponses des Ministres à ces questions, Paris 1950/51.

Journal Officiel de la République Française. Débats Parlementaires. Conseil de la République. Compte rendue in extenso des séances. Questions écrites et réponses des Ministres à ces questions, Paris 1951. 
Literaturverzeichnis

JOURNAL OFFICIEL, LOIS ET DÉCRETS
1948

KABINETTSPROTOKOLLE

KAISER, Brücke

LANDSBERG

LENZ, Zentrum

MCCLOY

MONTANMITBESTIMMUNG

NEUORDNUNG

PUBLIC PAPERS, TRUMAN 1945
Journal Officiel de la République Française. Lois et décrets, arrêtés, circulaires, avis, communications, informations et annonces. Jahrgang 1948, Paris 1948.

Die Kabinettsprotokolle der Bundesregierung, hrsg. für das Bundesarchiv von Hans Booms. Band 1: 1949, bearbeitet von Ulrich Enders und Konrad Reiser; Band 2: 1950, bearbeitet von Ulrich Enders und Konrad Kaiser; Band 3: 1950 Wortprotokolle, bearbeitet von Ulrich Enders und Konrad Kaiser; Band 4: 1951, bearbeitet von Ursula Hüllbusch; Band 5: 1952, bearbeitet von Kai von Jena, Boppard am Rhein 1982-1989.

Jakob Kaiser. Wir haben Brücke zu sein. Reden, Äußerungen und Aufsätze zur Deutschlandpolitik, hrsg. von Christian Hacke, Köln 1988.

Landsberg. Ein dokumentarischer Bericht, hrsg. vom Information Services Division Office of the U.S. High Commissioner for Germany, München 1951.

Im Zentrum der Macht. Das Tagebuch von Staatssekretär Lenz 1951-1953, bearbeitet von Klaus Gotto, Hans-Otto Kleinmann und Reinhard Schreiner, Düsseldorf 1989.

John J. McCloy. An American Architect of Postwar Germany. Profiles of a Transatlantic Leader and Communicator, hrsg. von Erika J. Fischer und Heinz-D. Fischer, Frankfurt am Main 1995.

Montanmitbestimmung. Das Gesetz über die Mitbestimmung der Arbeitnehmer in den Aufsichtsräten und Vorständen der Unternehmen des Bergbaus und der Eisen und Stahl erzeugenden Industrie vom 21. Mai 1951, bearbeitet von Gabriele Müller-List, Düsseldorf 1984.

Die Neuordnung der Eisen- und Stahlindustrie im Gebiet der Bundesrepublik Deutschland. Ein Bericht der Stahltreuhändervereinigung, München/Berlin 1954.

Public Papers of the Presidents of the United States. Harry S. Truman. Containing the Public Messages, Speeches, and Statements of the President, April 12 to December 31, 1945, Washington D.C. 1961. 
PUblic PAPERs, TRUMAN 1950

Public PaPers, TRUMAN 1951

LES RAPPORTS MENSUELS

REICHSGESETZBLATT

REUTER, Schriften-Reden

SAARPOLITIK

SCHUMACHER, Reden

SPEIDEL, Erinnerungen

STAATSVERTRAG

TEHERAN-JALTA-POTSDAM

Truman, Memoirs

UN GENERAL ASSEMBLY, OFFICIAL RECORDS, AD HOC POLITICAL COMMITTEE, 1951-52

UN GENERAL ASSEMBLY, OFFICLAL RECORDS, SIXTH SESSION ANNEXES, Agenda Item 65
Public Papers of the Presidents of the United States. Harry S. Truman. Containing the Public Messages, Speeches, and Statements of the President, January 1 to December 31, 1950, Washington D.C. 1965.

Public Papers of the Presidents of the United States. Harry S. Truman. Containing the Public Messages, Speeches, and Statements of the President, January 1 to December 31, 1951, Washington D.C. 1965.

Les Rapports Mensuels d'André FrançoisPoncet, Haut-Commissaire français en Allemagne 1949-1955, bearbeitet von Hans Manfred Bock, 2 Bände, Paris 1996.

Reichsgesetzblatt, hrsg. vom Reichsministerium des Innern, Berlin 1919-1945.

Ernst Reuter, Schriften-Reden. Band 4: Reden-Artikel-Briefe 1949 bis 1953 , bearbeitet von Hans J. Reichhardt, Berlin 1976.

Robert H. Schmidt, Saarpolitik 1945-1957. 2. Band: Entfaltung der Saarpolitik zwischen „Wirtschaftsanschlu $\beta^{\prime \prime}$ und „Europäisierung" 1945-1953, Berlin 1960.

Kurt Schumacher. Reden-Schriften-Korrespondenzen 1945-1952, hrsg. von Willy Albrecht, Berlin/Bonn 1985.

Hans Speidel, Aus unserer Zeit. Erinnerungen, 2. Auflage, Berlin/Frankfurt am Main/Wien 1977.

Gerald Stourzh, Geschichte des Staatsvertrages 1945-1955. Österreichs Weg zur Neutralität, 3. überarbeitete Auflage, Graz/Wien/Köln 1993.

Teheran-Jalta-Potsdam. Die sowjetischen Protokolle von den Kriegskonferenzen der ${ }_{n}$ Großen Drei“, hrsg. und eingeleitet von Alexander Fischer, 3. Auflage, Köln 1985.

Harry S. Truman. Memoirs. Band 2: Years of Trial and Hope, Garden City, N.Y. 1956.

United Nations. Official Records of the General Assembly. Sixth Session. Ad Hoc Political Committee, Summary Records of Meetings, 19 November to 31 January, 1951-52, Paris.

United Nations. Official Records of the General Assembly. Sixth Session Annexes, Palais de Chaillot, Paris, 1951-52. 


\section{UN GENERAL ASSEMBLY, OFFICIAL RECORDS, PLENARY MEETINGS 1951-52}

UN RESOLUTIONS I

UN RESOLUTIONS II

UNITED STATES. STATUTES AT LARGE 1948

UNTS

YEARBOOK OF THE UNITED NATIONS
United Nations. Official Records of the General Assembly, Sixth Session. Plenary Meetings, Verbatim Records of Meetings 6 November to 5 February, 1951-1952, Paris.

United Nations Resolutions. Series I: Resolutions Adopted by the General Assembly, hrsg. von Dusan J. Djonovich, New York 1972 ff.

United Nations Resolutions. Series II: Resolutions and Decisions Adopted by the Security Council, hrsg. von Dusan J. Djonovich, New York $1988 \mathrm{ff}$.

United States. Statutes at Large Containing the Laws and Concurrent Resolutions Enacted during the Second Session of the Eighteeth Congress of the United States of America 1948 and Proclamations, Treaties, and International Agreements other than Treaties. Band 62 in 3 Teilbänden, Washington D.C. 1949.

United Nations Treaty Series. Treaties and International Agreements Registered or Filed and Recorded with the Secretariat of the United Nations, [New York] 1946 ff.

Yearbook of the United Nations 1946/47, 1948/49, 1950 und 1951, hrsg. vom Department of Public Information United Nations, New York 1948-1952. 


\section{Abkürzungsverzeichnis}

\begin{tabular}{|c|c|c|c|}
\hline Abg. & Abgeordnete/Abgeordneter & EEC & $\begin{array}{l}\text { European Economic } \\
\text { Community }\end{array}$ \\
\hline $\begin{array}{l}\text { Abt. } \\
\text { a. D. }\end{array}$ & $\begin{array}{l}\text { Abteilung } \\
\text { außer Dienst }\end{array}$ & EGKS & $\begin{array}{l}\text { Europäische Gemeinschaft } \\
\text { für Kohle und Stahl }\end{array}$ \\
\hline AGSEC & Allied General Secretariat & EPU & European Payments Union \\
\hline $\begin{array}{l}\text { AHK } \\
\text { Ber. Nr. }\end{array}$ & $\begin{array}{l}\text { Alliierte Hohe Kommission } \\
\text { Bericht-Nummer }\end{array}$ & EVG & $\begin{array}{l}\text { Europäische Verteidigungs- } \\
\text { gemeinschaft }\end{array}$ \\
\hline BHE & $\begin{array}{l}\text { Block der Heimatvertrie- } \\
\text { benen und Entrechteten }\end{array}$ & FAO & $\begin{array}{l}\text { Food and Agriculture } \\
\text { Organization of the } \\
\text { United Nations }\end{array}$ \\
\hline $\begin{array}{l}\text { Bibl. } \\
\text { BND }\end{array}$ & $\begin{array}{l}\text { Bibliothek } \\
\text { Bundesnachrichtendienst }\end{array}$ & FDJ & Freie Deutsche Jugend \\
\hline BSt & Besatzungsstatut & FDP & $\begin{array}{l}\text { Freie Demokratische } \\
\text { Partei }\end{array}$ \\
\hline $\begin{array}{l}\text { BT } \\
\text { CDU }\end{array}$ & $\begin{array}{l}\text { Bundestag } \\
\text { Christlich-Demokratische }\end{array}$ & GARIOA & $\begin{array}{l}\text { Government and Relief in } \\
\text { Occupied Areas }\end{array}$ \\
\hline & Union Deutschlands & Geh./geh. & Geheim/geheim \\
\hline ČsR & Československá Republika & GG & Grundgesetz \\
\hline $\begin{array}{l}\text { CSU } \\
\text { DDR }\end{array}$ & $\begin{array}{l}\text { Christlich-Soziale Union } \\
\text { Deutsche Demokratische }\end{array}$ & $\mathrm{HaPol}$ & $\begin{array}{l}\text { Handelspolitische } \\
\text { Abteilung }\end{array}$ \\
\hline Del. & $\begin{array}{l}\text { Republik } \\
\text { Delegation }\end{array}$ & HICOG & $\begin{array}{l}\text { United States High } \\
\text { Commissioner for Germany }\end{array}$ \\
\hline DGB & $\begin{array}{l}\text { Deutscher Gewerkschafts- } \\
\text { bund }\end{array}$ & HICOM & $\begin{array}{l}\text { United States High } \\
\text { Commission for Germany }\end{array}$ \\
\hline DKBL & $\begin{array}{l}\text { Deutsche Kohlenbergbau- } \\
\text { Leitung }\end{array}$ & IBFG & $\begin{array}{l}\text { Internationaler Bund } \\
\text { Freier Gewerkschaften }\end{array}$ \\
\hline DKV & $\begin{array}{l}\text { Deutsche Kohle-Verkaufs- } \\
\text { organisation }\end{array}$ & ICAO & $\begin{array}{l}\text { International Civil } \\
\text { Aviation Organization }\end{array}$ \\
\hline $\begin{array}{l}\text { DP } \\
\text { DPA }\end{array}$ & Deutsche Partei & ILO & $\begin{array}{l}\text { International Labour } \\
\text { Organization }\end{array}$ \\
\hline DPS & Demokratische Partei Saar & IRO & $\begin{array}{l}\text { International Refugee } \\
\text { Organization }\end{array}$ \\
\hline ECA & $\begin{array}{l}\text { Economic Cooperation } \\
\text { Administration }\end{array}$ & IWF & $\begin{array}{l}\text { Internationaler Währungs- } \\
\text { fonds }\end{array}$ \\
\hline ECE & $\begin{array}{l}\text { Economic Commission for } \\
\text { Europe }\end{array}$ & JELA & $\begin{array}{l}\text { Joint Export-Import } \\
\text { Agency }\end{array}$ \\
\hline Ecosoc & $\begin{array}{l}\text { Economic and Social } \\
\text { Council }\end{array}$ & JRSO & $\begin{array}{l}\text { Jewish Restitution } \\
\text { Successor Organization }\end{array}$ \\
\hline EDC & $\begin{array}{l}\text { European Defense } \\
\text { Community }\end{array}$ & Kontr. Nr. & Kontroll-Nummer \\
\hline
\end{tabular}




\begin{tabular}{|c|c|c|c|}
\hline KPD & $\begin{array}{l}\text { Kommunistische Partei } \\
\text { Deutschlands }\end{array}$ & SHAPE & $\begin{array}{l}\text { Supreme Headquarters } \\
\text { Allied Powers Europe }\end{array}$ \\
\hline Kult. & Kulturabteilung & SPD & $\begin{array}{l}\text { Sozialdemokratische Partei } \\
\text { Deutschlands }\end{array}$ \\
\hline $\mathrm{KZ}$ & Konzentrationslager & & Sozialistische Reichsnartei \\
\hline LR I & Legationsrat I. Klasse & SKF & Soziansiscne Keichsparter \\
\hline MB & Ministerbüro & SS & Schutzstaffel \\
\hline MD & Ministerialdirektor & StEG & $\begin{array}{l}\text { Staatliche Erfassungs- } \\
\text { Gesellschaft für öffent- }\end{array}$ \\
\hline $\mathrm{MdB}$ & Mitglied des Bundestages & & liches Gut \\
\hline MDg & Ministerialdirigent & StS & Staatssekretär \\
\hline MEZ & Mitteleuropäische Zeit & TCC & $\begin{array}{l}\text { Temporary Council } \\
\text { Committee }\end{array}$ \\
\hline Mio. & Million/en & Tgb. Nr. & Tagebuch-Nummer \\
\hline MR & Ministerialrat & TOA & Tarifordnung für \\
\hline Mrd. & Milliarde/n & & Angestellte \\
\hline MRP & $\begin{array}{l}\text { Mouvement Républicain } \\
\text { Populaire }\end{array}$ & UdSSR & $\begin{array}{l}\text { Union der Sozialistischen } \\
\text { Sowjetrepubliken }\end{array}$ \\
\hline NATO & North Atlantic Treaty & UK & United Kingdom \\
\hline & & UN & United Nations \\
\hline OEEC & $\begin{array}{l}\text { Organisation for European } \\
\text { Economic Cooperation }\end{array}$ & UNESCO & $\begin{array}{l}\text { United Nations Educa- } \\
\text { tional, Scientific and }\end{array}$ \\
\hline ÖVP & Österreichische Volks- & & Cultural Organization \\
\hline Or & & UNHCR & $\begin{array}{l}\text { United Nations High } \\
\text { Commissioner for Refugees }\end{array}$ \\
\hline ORR & Oberregierungsrat & UNO & $\begin{array}{l}\text { United Nations } \\
\text { Organization }\end{array}$ \\
\hline OSS & Office of Strategic Services & US & United States \\
\hline Prot. & Protokoll & USA & United States of America \\
\hline $\mathrm{RM}$ & Reichsmark & VLR & Vortragender Legationsrat \\
\hline R.P.F. & $\begin{array}{l}\text { Rassemblement du Peuple } \\
\text { Français }\end{array}$ & VN & Vereinte Nationen \\
\hline $\mathrm{RR}$ & Regierungsrat & VS & Verschlußsache \\
\hline SACEUR & $\begin{array}{l}\text { Supreme Allied } \\
\text { Commander Europe }\end{array}$ & WAV & $\begin{array}{l}\text { Wirtschaftliche Aufbau- } \\
\text { vereinigung }\end{array}$ \\
\hline SD & Sicherheitsdienst & WHO & World Health Organization \\
\hline SED & $\begin{array}{l}\text { Sozialistische Einheits- } \\
\text { partei Deutschlands }\end{array}$ & $\begin{array}{l}\text { W.K. } \\
\text { z.Wv. }\end{array}$ & $\begin{array}{l}\text { Wehrkreis } \\
\text { zur Wiederverwendung }\end{array}$ \\
\hline
\end{tabular}

